\title{
LISTADO ACTUALIZADO DE ESPECIES DE ARANEIDOS Y TOMÍSIDOS (ARANEAE, ARANEIDAE Y THOMISIDAE) DE LA COMUNIDAD DE MADRID: MAPAS DE DISTRIBUCIÓN CONOCIDA, POTENCIAL Y PATRONES DE RIQUEZA
}

\author{
A. Jiménez-Valverde ${ }^{1}$, J. M. Lobo ${ }^{1}$ y M. L. López Martos ${ }^{1}$
}

\begin{abstract}
RESUMEN
Se actualiza el listado de especies de las familias Araneidae y Thomisidae de la Comunidad de Madrid, citando 3 especies nuevas de araneidos y 15 de tomísidos, con lo que el número de especies de la primera familia asciende a 30 y a 43 el de la segunda. Se eliminan del catálogo Heriaeus hirtus y Larinioides cornutus. Tras elaborar los mapas de distribución conocida y potencial se constata una falta de datos corológicos, especialmente acusado en la familia Thomisidae debido a su carácter críptico. Los mapas de riqueza específica reflejan un patrón común, con valores máximos de riqueza en la Rampa y valores mínimos en el sur de la Comunidad y en las zonas de alta montaña. Aunque los valores brutos de riqueza sean, seguramente, una subestimación de los valores reales, el patrón general puede ser explicado en base a las preferencias ecológicas de las dos familias y al patrón espacial de la alteración antrópica del hábitat en la provincia de Madrid. Se resalta la necesidad urgente de efectuar muestreos para completar los grandes huecos de información.

Palabras clave: Araneae, Araneidae, Thomisidae, Comunidad de Madrid, catálogo, mapas de distribución, distribución potencial, riqueza de especies.
\end{abstract}

\section{ABSTRACT \\ Updated checklist of araneid and tomisid (Araneae, Araneidae and Thomisidae) species of Comunidad de Madrid: known and potential distribution maps, and richness patterns}

The list of species of the families Araneidae and Thomisidae in Comunidad de Madrid is updated. 3 new species of Araneidae and 15 of Thomisidae are added to the list, for a total of 30 species in the first family, and of 46 in the second. Heriaeus hirtus and Larinioides cornutus are eliminated from the catalogue. After the distributional and predictive maps were drawn, an significant lack of distributional records is noticed, especially in the Thomisidae due, probably, to their cryptic nature. Richness maps show a common pattern, with maximum values in the Rampa and minimum ones in the south of the province and high altitudes of the Sierra. Although richness scores are probably a sub estimation of true values, the general pattern can be explained according to the ecological preferences of the two families and the spatial pattern of anthropogenic alteration of the habitat in Madrid. The imperative necessity of making field surveys to complete data gaps is highlighted.

Key words: Araneae, Araneidae, Thomisidae, Comunidad de Madrid, checklist, distributional maps, potential distribution, species richness.

Departamento de Biodiversidad y Biología Evolutiva, Museo Nacional de Ciencias Naturales (CSIC), c/ José Gutiérrez Abascal 2, 28006 Madrid, España. ${ }^{1}$ Tel.: 914111328 ext.1212; e-mail: mcnaj651@mncn.csic.es 


\section{Introducción}

El orden Araneae comprende 36.000 especies aproximadamente, aunque se estima que su número debe ser bastante mayor (Coddington \& Levi, 1991). Debido a su condición de depredadores generalistas y ubiquistas, las arañas juegan un importante papel en los sistemas ecológicos (Wise, 1993) y ofrecen un gran potencial para la regulación de las poblaciones de artrópodos (Marc et al., 1999). La familia Araneidae es una de las más exitosas (aproximadamente 2.600 especies descritas; Foelix, 1996), integrada por especies que construyen telas orbiculares para la captura de sus presas. Por el contrario, las arañas de la familia Thomisidae no emplean telas para la captura de sus presas, sino que permanecen al acecho sobre hojas y flores, pasando inadvertidas gracias a su coloración críptica. Algunos géneros, como Xysticus y Ozyptila, capturan a sus presas entre la hojarasca y la vegetación herbácea.

En la Península Ibérica hay citadas, hasta el momento, 1210 especies de arañas, aunque indudablemente su número crecerá a medida que aumenten los estudios taxonómicos y faunísticos. La familia Araneidae cuenta con 56 especies repartidas en 22 géneros, mientras que la familia Thomisidae tiene 66 especies en 14 géneros. La ausencia de tradición aracnológica en la Península Ibérica ha provocado que el conocimiento actual de su aracnofauna sea bastante limitado; los catálogos son escasos y la mayoría de las citas antiguas, muchas de ellas erróneas o, cuanto menos, dudosas, tal y como manifiestan Melic (2001) y Morano (2004). Además, la ausencia y dispersión de la bibliografía necesaria para efectuar correctas identificaciones no facilita la labor de estudio. La Comunidad de Madrid no es ajena a este desconocimiento general, contando únicamente con un catálogo actualizado de la fauna de la familia Salticidae (Jiménez-Valverde, 2005). Este trabajo pretende elaborar un listado actualizado de las familias Araneidae y Thomisidae para la provincia, recopilando las citas existentes y aportando nuevos datos de campo. Con esta información se han elaborado mapas preliminares de distribución potencial para cada especie y un mapa de riqueza para cada una de las dos familias con la intención de iniciar una primera aproximación al estudio de los patrones de diversidad de arañas en la Comunidad de Madrid.

\section{Material y métodos}

Para la elaboración del listado de especies se ha tenido en cuenta la información bibliográfica pre- viamente existente compilada por Eduardo Morano en Aproximación al Catálogo de Arañas Ibero Baleares (disponible en http://perso.wanadoo. es/catalogoaranas), se ha revisado la bibliografía más reciente no tenida en cuenta por este autor y la tesina de M. F. Camargo (1982), se ha revisado la colección del Museo Nacional de Ciencias Naturales de Madrid y la colección particular del primer autor (AJV), y se ha incluido el material colectado por éste durante la realización de su tesis doctoral. Durante este último estudio, AJV realizó muestreos primaverales intensivos según el protocolo desarrollado por Jiménez-Valverde \& Lobo (2005), específicamente desarrollado para el inventariado de las familias Araneidae y Thomisidae. Este protocolo supone el empleo de tres técnicas complementarias de captura (mangueo, batido y trampas pitfall) en un área de $1 \mathrm{~km}^{2}$. Las localidades de muestreo se seleccionaron siguiendo una metodología para representar lo más fielmente posible la diversidad ambiental y espacial de la región (ver Jiménez-Valverde \& Lobo, 2004).

El área sobre la cual se han realizado los mapas de distribución potencial es un rectángulo de $27.200 \mathrm{~km}^{2}$ (160 km x $170 \mathrm{~km}$ ) que abarca, no solo todo el territorio de la Comunidad de Madrid, sino también parte de las provincias limítrofes de Ávila, Cuenca, Guadalajara, Segovia, Toledo y Valladolid. Los cuatro vértices de este área son los correspondientes a las cuadrículas UTM de $100 \mathrm{~km}^{2}$ 30TUL56, 30TWL06, 30SUK60 y 30SWK00. Tras compilar todas las citas de estas dos familias de arañas en la Comunidad de Madrid (ver Apéndice 1), se procedió a elaborar mapas de distribución para cada una de las especies y también a estimar su distribución potencial. Si los factores históricos únicos e irrepetibles no han influenciado la distribución de las especies o si la extensión del área considerada no es extensa, la distribución potencial de una especie puede asumirse como aquella formada por el conjunto de localidades con condiciones ambientales similares a las existentes en los puntos de presencia conocidos (ver, por ejemplo, Busby, 1986). Para cada una de las especies la hipótesis de distribución potencial se estimó teniendo en cuenta la información de cuatro variables climáticas: precipitación total anual, precipitación durante el estío (meses Junio, Julio y Agosto), temperatura máxima del mes más calido (Julio) y temperatura mínima del mes más frío (Enero). Tras calcular los valores máximos y mínimos de cada una de estas variables climáticas para aquellas cuadrículas de $1 \mathrm{~km}^{2}$ en las que ha sido observada la especie (en el caso de que las citas estuviesen referidas a cuadrículas de 10 
$\mathrm{km}^{2}$ se tomó el centroide), se determinaron como lugares potencialmente favorables las regiones con valores climáticos situados entre ambos extremos. La cartografía climática fue proporcionada por el Instituto Nacional de Meteorología, siendo elaborada mediante la utilización de un Sistema de Información Geográfico (Idrisi Kilimanjaro; Clark Labs., 2003).

\section{Listado de especies}

ARANEIDAE Latreille, 1806

Aculepeira Chamberlin \& Ivie, 1942 Aculepeira armida (Audouin, 1826) Aculepeira ceropegia (Walckenaer, 1802)

Agalenatea Archer, 1951

Agalenatea redii (Scopoli, 1763)

Araneus Clerck, 1758

Araneus angulatus Clerck, 1758

Araneus diadematus Clerck, 1758

Araneus pallidus (Olivier, 1789)

Araniella Chamberlin \& Ivie, 1942

Araniella alpica (Koch, L., 1869)

Araniella cucurbitina (Clerck, 1758)

Araniella inconspicua (Simon, 1874)

Araniella opisthographa (Kulczynski, 1905)

Argiope Audouin, 1826

Argiope bruennichi (Scopoli, 1772)

Argiope lobata (Pallas, 1772)

Atea Koch, C.L., 1837

Atea sturmi (Hahn, 1831)

Cercidia Thorell, 1869

Cercidia prominens (Westring, 1851)

Cyclosa Menge, 1866

Cyclosa algerica Simon, 1885

Cyclosa conica (Pallas, 1772)

Cyclosa insulana (Costa, 1834)

Gibbaranea Archer, 1951

Gibbaranea bituberculata (Walckenaer, 1802)

Gibbaranea gibbosa (Walckenaer, 1802)

Hypsosinga Ausserer, 1871

Hypsosinga albovittata (Westring, 1851)

Hypsosinga pygmaea (Sundevall, 1831)

Hypsosinga sanguinea (Koch, C.L., 1844)

Larinioides Caporiacco, 1934

Larinioides sclopetarius (Clerck, 1758)

Larinioides suspicax (Pickard-Cambridge, O., 1876)

Mangora Pickard-Cambridge, O., 1889

Mangora acalypha (Walckenaer, 1802)

Neoscona Simon, 1864

Neoscona adianta (Walckenaer, 1802)

Neoscona subfusca (Koch, C.L., 1837)

Singa Koch, C.L., 1836

Singa hamata (Clerck, 1758)

Zilla Koch, C.L., 1834

Zilla diodia (Walckenaer, 1802)
Zygiella Pickard-Cambridge, F.O., 1902

Zygiella $x$-notata (Clerck, 1758)

THOMISIDAE Sundevall, 1833

Coriarachne Thorell, 1870

Coriarachne sp.

Firmicinus Simon, 1895

Firmicinus bivittatus Simon, 1895

Diaea Thorell, 1869

Diaea dorsata (Fabricius, 1777)

Heriaeus Simon, 1875

Heriaeus mellotei Simon, 1886

Misumena Latreille, 1804

Misumena vatia (Clerck, 1758)

Misumenops Pickard-Cambridge, F.O., 1900

Misumenops sp.

Ozyptila Simon, 1864

Ozyptila atomaria (Panzer, 1801)

Ozyptila bicuspis Simon, 1932

Ozyptila blackwalli Simon, 1875

Ozyptila pauxilla (Simon, 1870)

Ozyptila perplexa Simon, 1875

Ozyptila umbraculorum Simon, 1932

Pistius Simon, 1875

Pistius truncatus (Pallas, 1772)

Runcinia Simon, 1875

Runcinia grammica (Koch, C.L., 1837)

Synema Simon, 1864

Synaema globosum (Fabricius, 1775)

Thomisus Walckenaer, 1805

Thomisus onustus Walckenaer, 1805

Tmarus Simon, 1875

Tmarus piochardi (Simon, 1866)

Tmarus staintoni (Pickard-Cambridge, O., 1873)

Tmarus stellio Simon, 1875

Xysticus Koch, C.L., 1835

Xysticus acerbus Thorell, 1872

Xysticus audax (Schrank, 1803)

Xysticus bifasciatus Koch, C.L., 1837

Xysticus bliteus (Simon, 1875)

Xysticus bufo (Dufour, 1820)

Xysticus caperatus Simon, 1875

Xysticus cor Canestrini, 1873

Xysticus cribatus Simon, 1885

Xysticus cristatus (Clerck, 1758)

Xysticus erraticus (Blackwall, 1834)

Xysticus ferrugineus Menge, 1876

Xysticus gallicus Simon, 1875

Xysticus grallator Simon, 1932

Xysticus kempelini Thorell, 1872

Xysticus kochi Thorell, 1872

Xysticus lanio Koch, C.L., 1835

Xysticus lineatus (Westring, 1851)

Xysticus ninni Thorell, 1872

Xysticus nubilus Simon, 1875

Xysticus ovatus Simon, 1876

Xysticus robustus (Hahn, 1832)

Xysticus sabulosus (Hahn, 1832)

Xysticus semicarinatus Simon, 1932

Xysticus ulmi (Hahn, 1832) 
Comentarios al listado: especies nuevas y de interés para la fauna ibérica y madrileña y especies dudosas

Se conocen 30 especies de araneidos y 43 de tomísidos para la Comunidad de Madrid, lo que representa el 54 y el $65 \%$, respectivamente, de la fauna Ibérica. En la Tabla 1 se muestran las 18 nuevas citas para la Comunidad de Madrid aparecidas tras la revisión del material depositado en la colección del Museo Nacional de Ciencias Naturales y las colectas efectuadas por el primer autor. Quince de estas especies corresponden a la familia Thomisidae, mientras que las 3 restantes corresponden a la familia Araneidae. Se cita por primera vez para la Península Ibérica a $X$. ovatus, especie hasta ahora únicamente conocida de Francia (Platnick, 2005). De especial interés resulta la cita de $A$. inconspicua, ya que es una especie bastante desconocida en la Península Ibérica hasta ahora citada únicamente del norte de España (sin especificar localidad) y de Retuerta del Bullaque, Ciudad Real (Jiménez-Valverde et al., 2004). Igualmente,
F. bivittatus sólo se conocía de La Granja, Segovia (Simon, 1932). Tmatus stellio fue citada por primera vez para la Península Ibérica en De Castro \& Alberdi (2002) a partir de un ejemplar colectado en Navarra, por lo que la localidad que se aporta aquí es la segunda cita de la especie en la Península y amplia su rango de distribución conocido. X. bifasciatus solamente ha sido reportado de Gerona (Fernández Galiano, 1910) y Portugal (Cardoso, 2000), por lo que en este trabajo se cita por segunda vez para España y se aumenta su rango de distribución. También se aumenta el área de distribución conocida para $X$. lineatus, especie hasta ahora sólo citada de Huesca (Melic, 2000) y Portugal (Cardoso, 2000).

La Dra. Carmen Urones, tras revisar el material reportado para Madrid e identificado como Heriaeus hirtus (Latreille, 1819) por Fernández Galiano (1910), llega a la conclusión de que no puede asignarse con seguridad a esta especie ya que se trata de individuos juveniles, etiquetándolos como Heriaeus sp., no debiendo H. hirtus ser

Tabla 1.- Especies con nuevas citas para la Península Ibérica y/o para la Comunidad de Madrid, indicando la fuente del registro (MNCN, colección del Museo Nacional de Ciencias Naturales; AJV, colección particular del primer autor; MtAJV, muestreos de campo de la tesis doctoral del primer autor).

Table 1.- Species with new citations for the Iberian Peninsula and/or for Comunidad de Madrid, indicating the source of the record (MNCN, collection of the National Museum of Natural Sciences; AJV, private collection of the first author; MtAJV, field samplings of the first author).

\begin{tabular}{|c|c|c|c|c|c|}
\hline \multirow[t]{2}{*}{ Especies } & \multirow{2}{*}{$\begin{array}{c}\text { Nueva cita } \\
\text { Península Ibérica }\end{array}$} & \multirow{2}{*}{$\begin{array}{c}\text { Nueva cita } \\
\text { Comunidad de Madrid }\end{array}$} & \multicolumn{3}{|c|}{ Fuente } \\
\hline & & & MNCN & AJV & MtAJV \\
\hline \multicolumn{6}{|l|}{ Araneidae } \\
\hline Araniella inconspicua & & - & & & - \\
\hline Atea sturmi & & - & & & - \\
\hline Zygiella $x$-notata & & - & & - & - \\
\hline \multicolumn{6}{|l|}{ Thomisidae } \\
\hline Diaea dorsata & & - & & & - \\
\hline Firmicinus bivittatus & & - & - & & \\
\hline Misumenops sp. & & - & & & - \\
\hline Ozyptila atomaria & & - & & & - \\
\hline Pistus truncatus & & - & - & & - \\
\hline Tmatus stellio & & - & & & - \\
\hline Xysticus bifasciatus & & - & & - & \\
\hline Xysticus cristatus & & - & - & & - \\
\hline Xysticus erraticus & & - & - & & - \\
\hline Xysticus gallicus & & - & & & - \\
\hline Xysticus kempelini & & - & & & - \\
\hline Xysticus lineatus & & - & - & & \\
\hline Xysticus ninni & & - & & & - \\
\hline Xysticus ovatus & - & - & - & & \\
\hline Xysticus robustus & & - & & - & \\
\hline
\end{tabular}




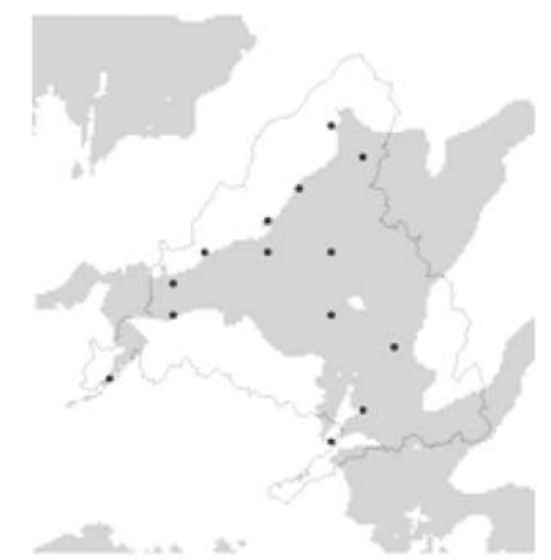

Aculepeira armida

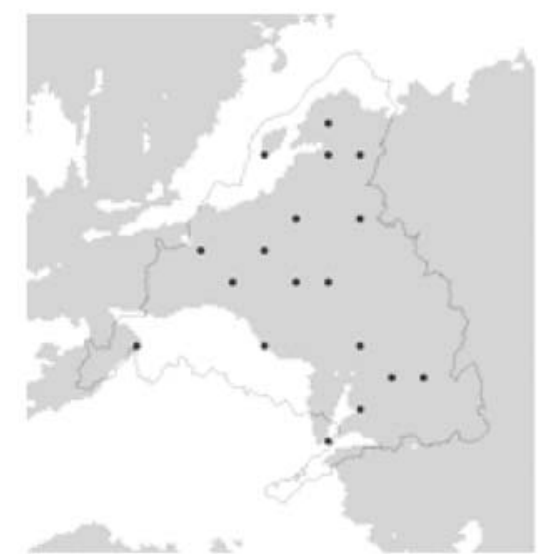

Agalenatea redif

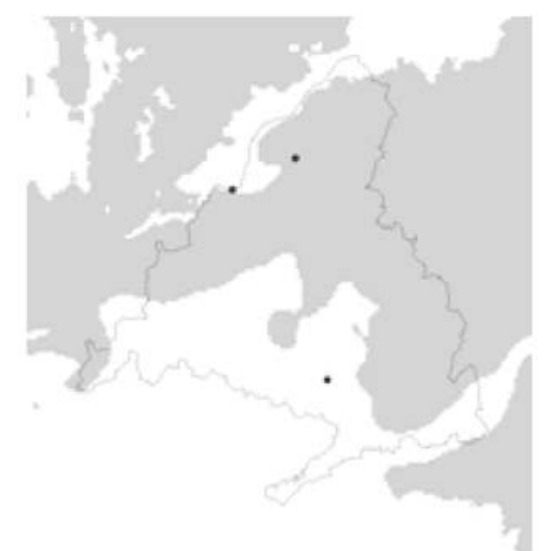

Araneus diadematus

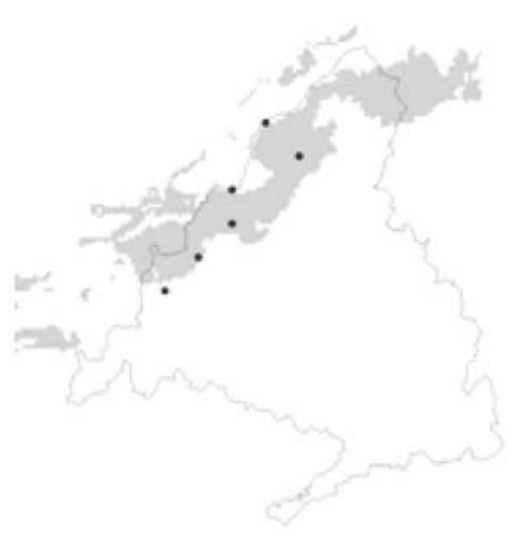

Aculepeira ceropegia

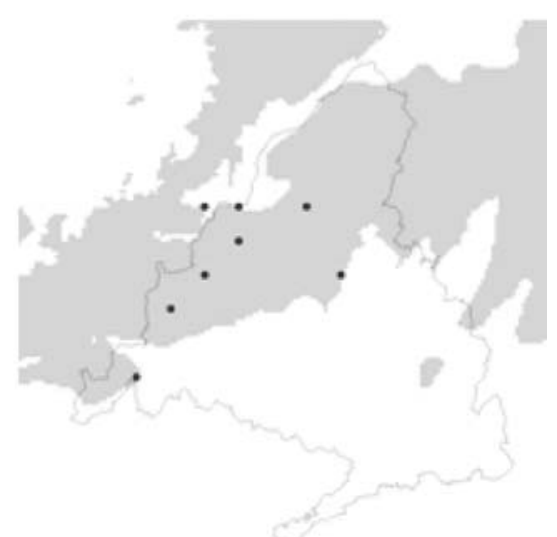

Araneus angulatus

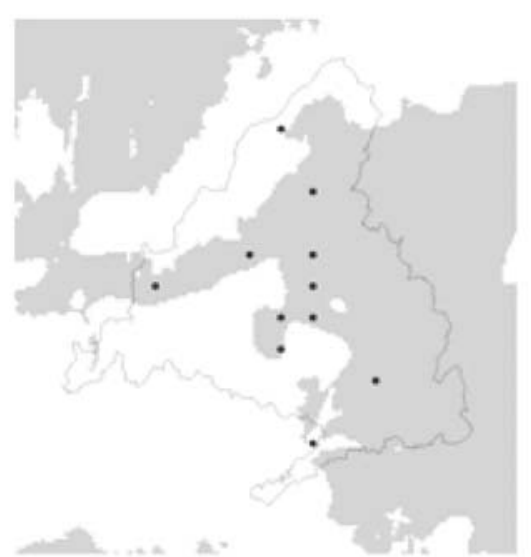

Araneus pallidus

Fig. 1.- Mapas de distribución conocida y potencial de las especies de la familia Araneidae de la Comunidad de Madrid.

Fig. 1.- Known and potential distribution maps of the species of the family Araneidae in Comunidad de Madrid. 


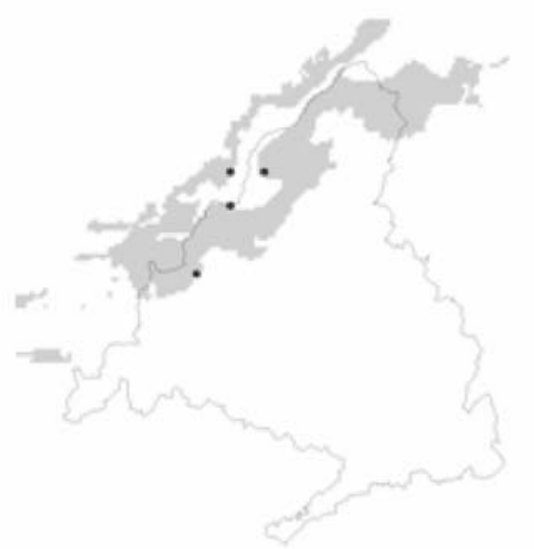

Araniella alpica

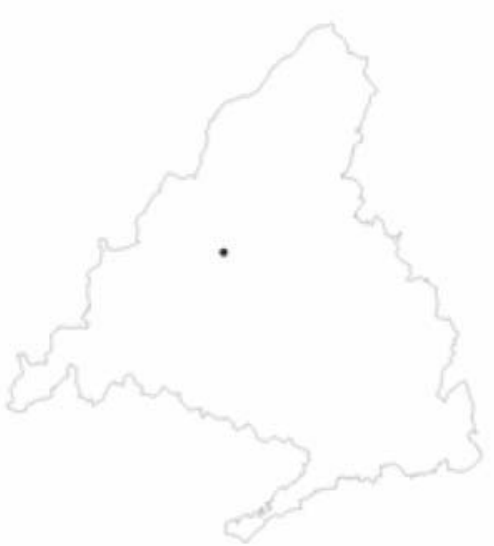

Araniella inconspicua

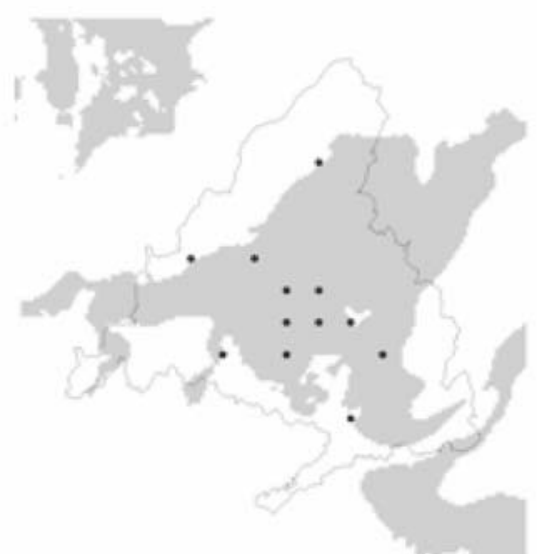

Argiope bruennichi

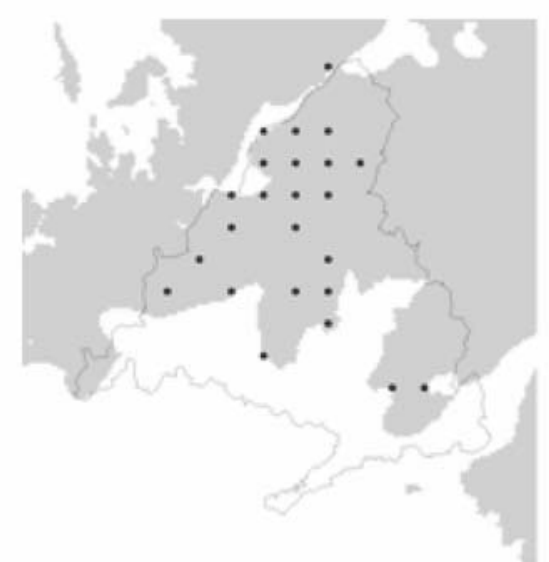

Araniella cucurbitina

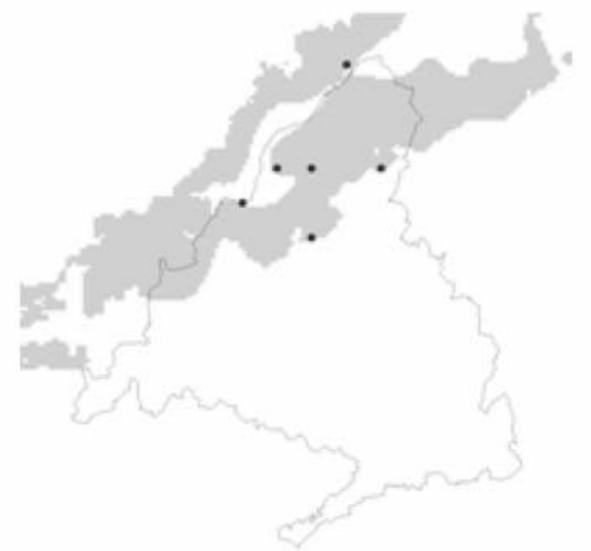

Araniella opisthographa

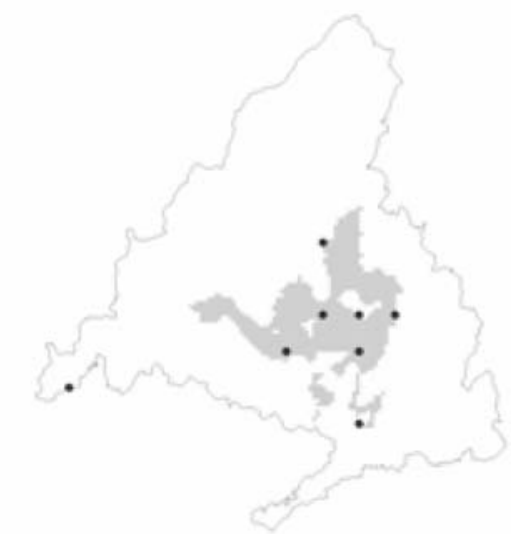

Argiope lobata 


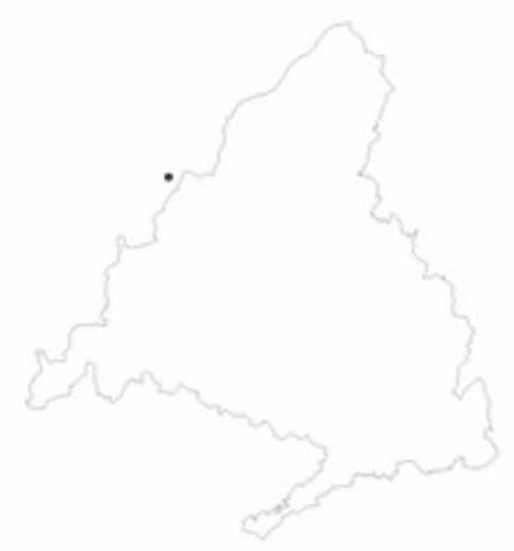

Atea sturmi

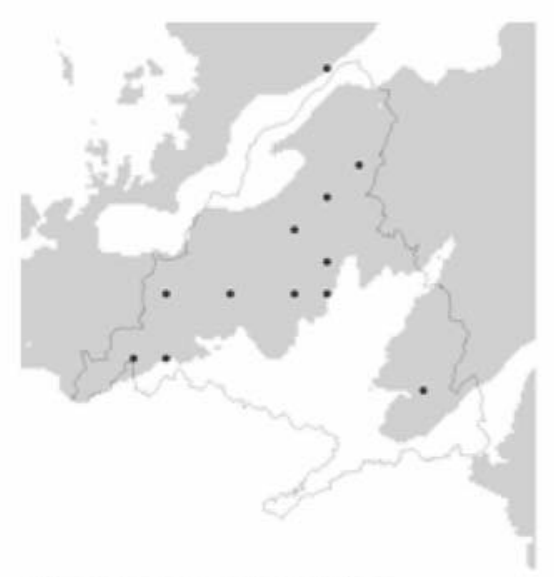

Cyclosa algerica

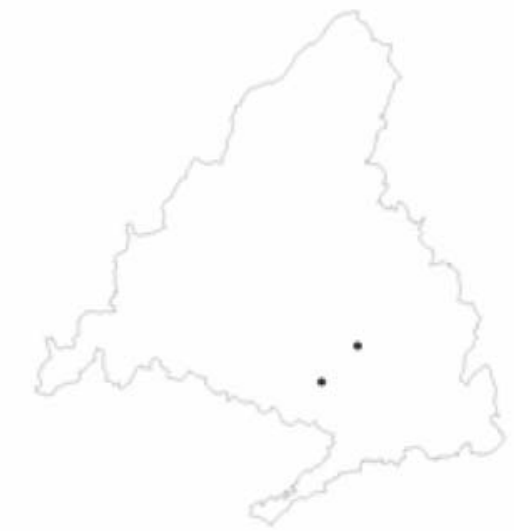

Cyclosa insulana

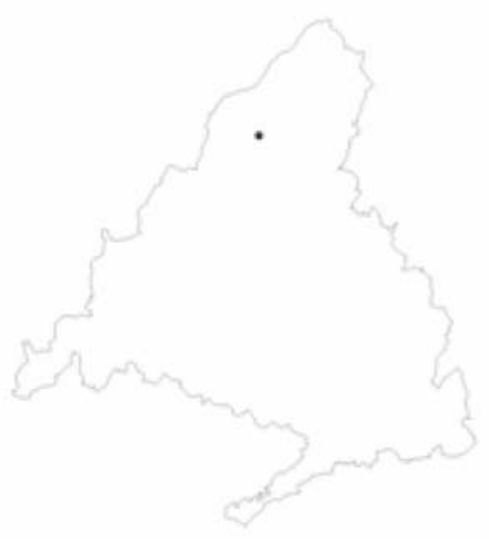

Cercidia prominens

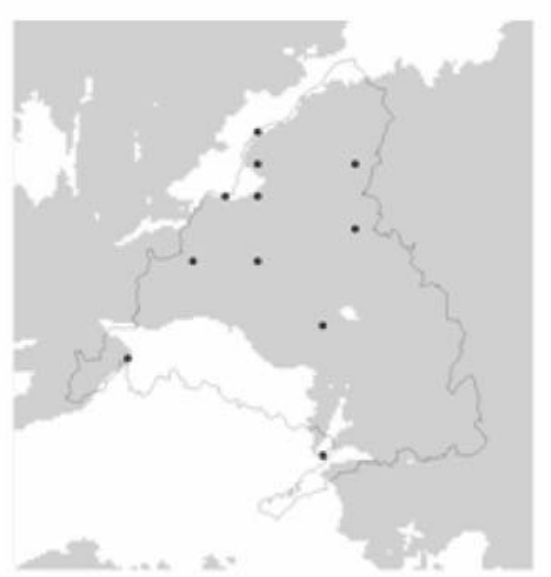

Cyclosa conica

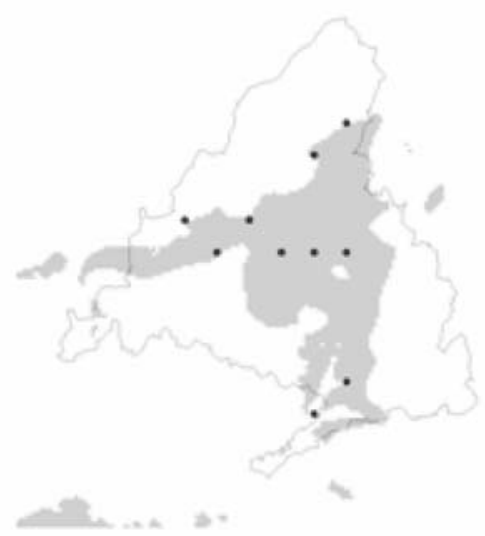

Gibbaranea bituberculata 


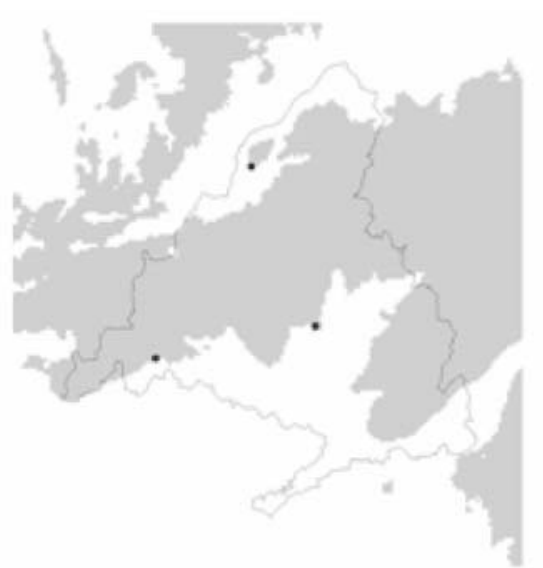

Gibbaranea gibbosa

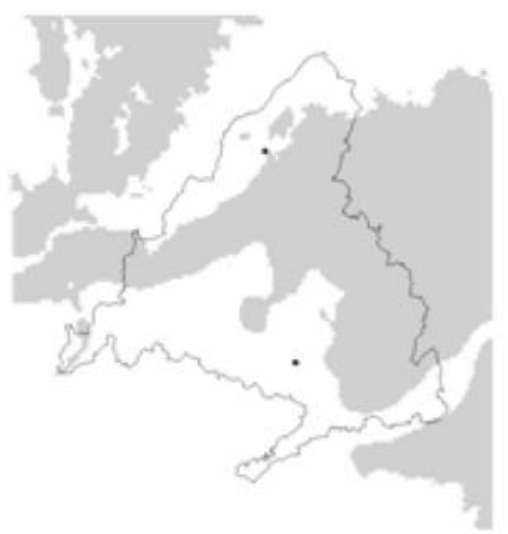

Hypsosinga pygmaea

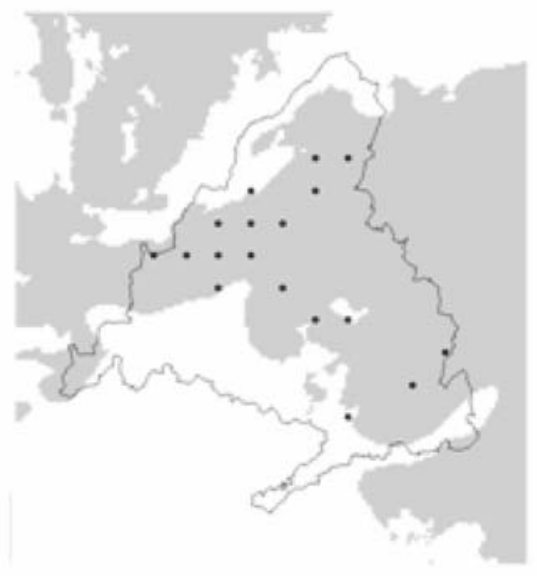

Larinioides sclopetarius

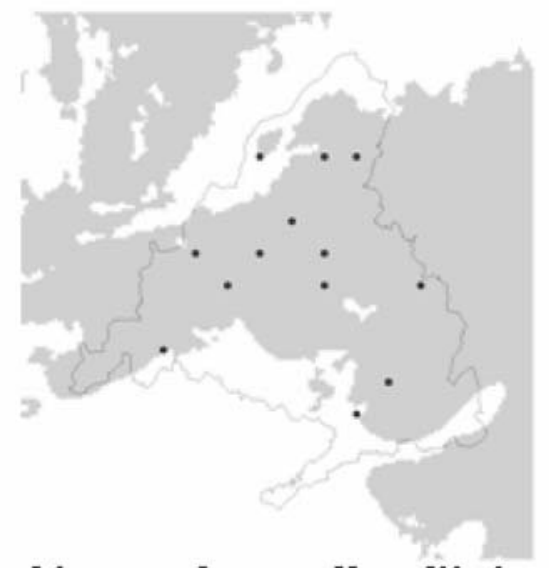

Hypsosinga albovittata

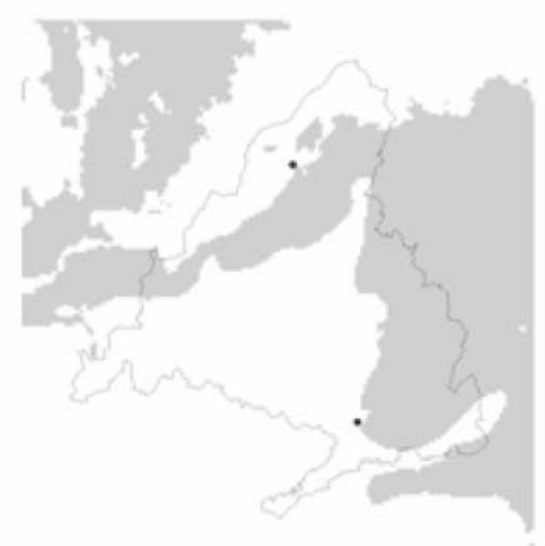

Hypsosinga sanguinea

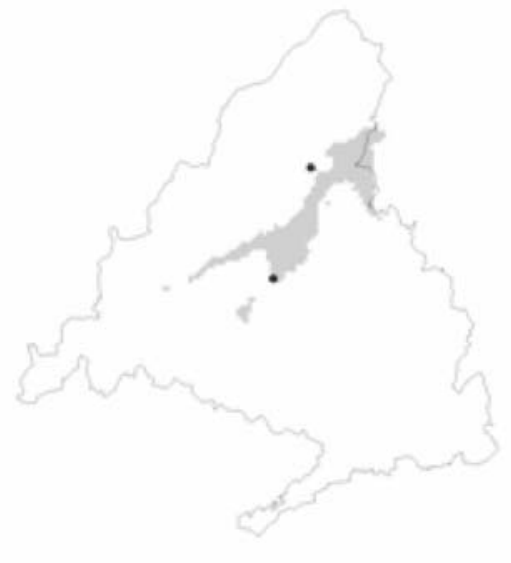

Larinioides suspicax 


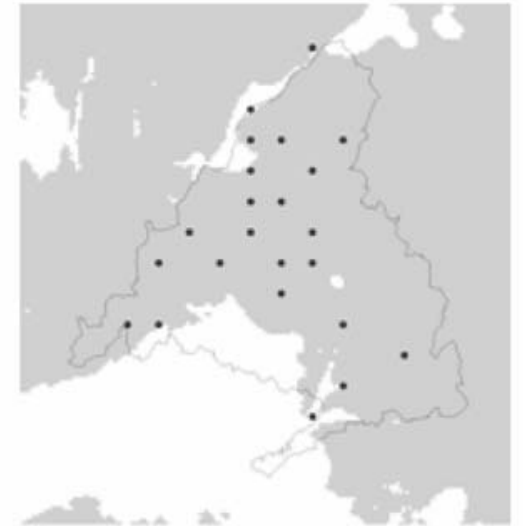

Mangora acalypha

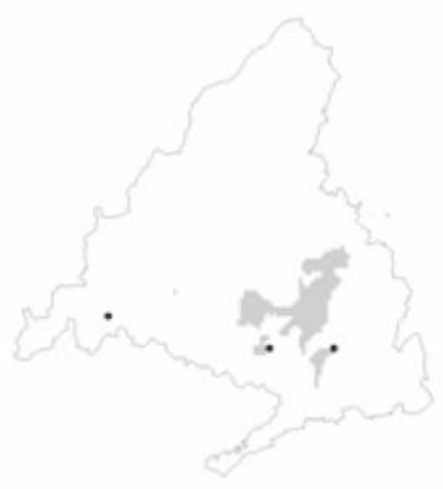

Neoscona subfusca

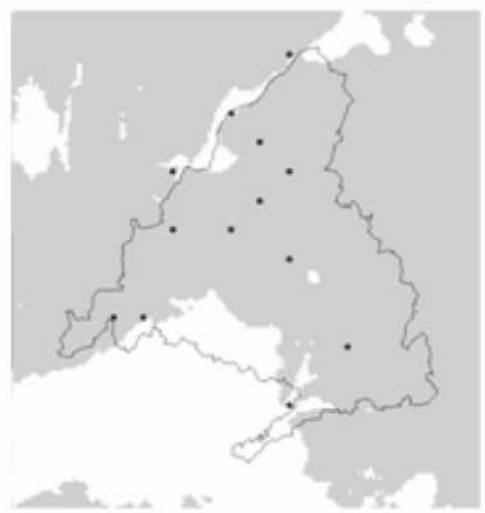

Zilla diodia

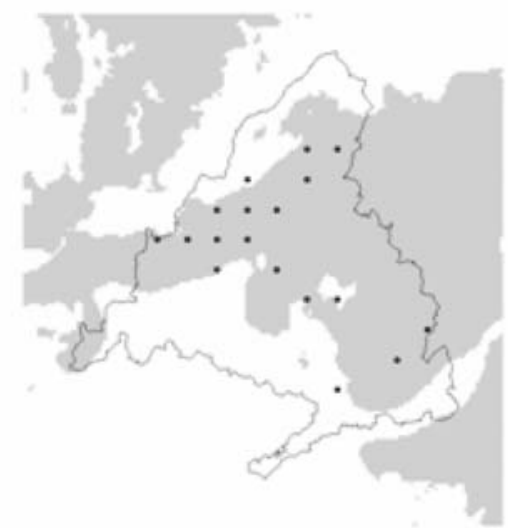

Neoscona adianta

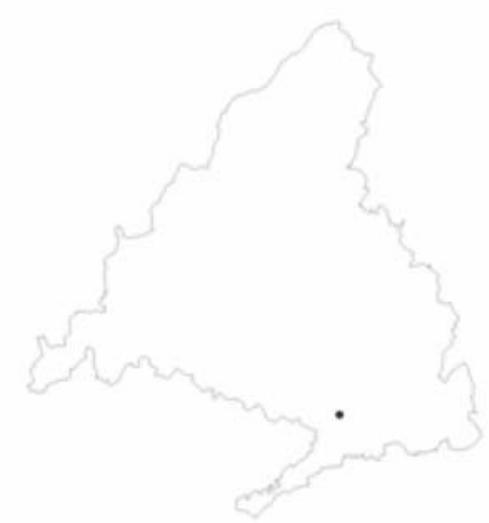

Singa hamata

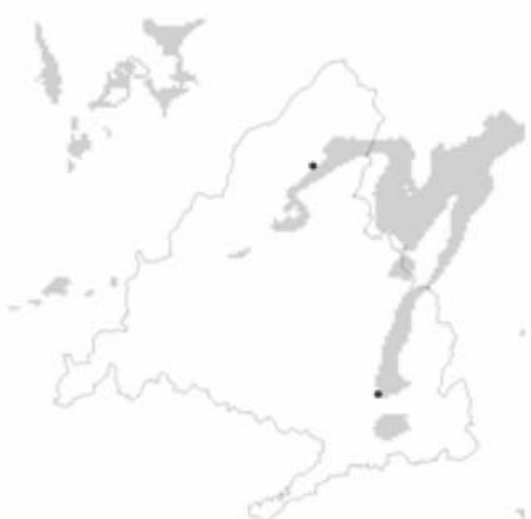

Zygiella x-notata

Fig. 1.-Fin. 


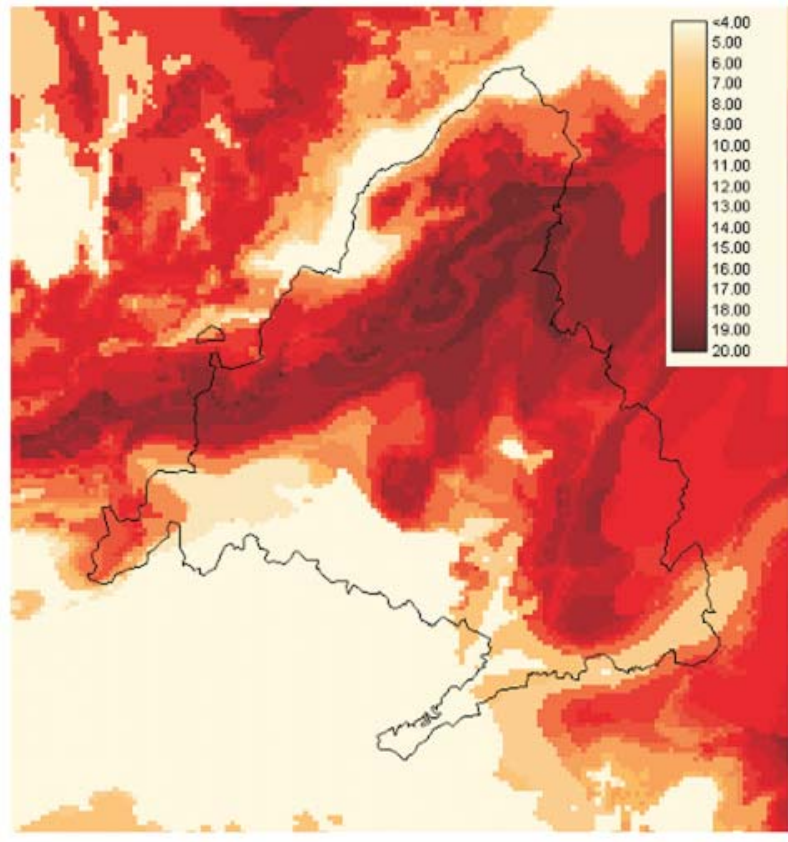

Riqueza de la familia Araneidae

Fig. 2.- Mapa de riqueza predicha de la familia Araneidae en la Comunidad de Madrid. Este mapa es el resultado de la suma de los mapas potenciales individuales de distribución.

Fig. 2.- Predicted species richness of the family Araneidae in Comunidad de Madrid, calculated as the sum of individual potential distribution maps.

incluida en el elenco de especies de la Comunidad de Madrid. Tampoco hemos considerado las citas de Larinioides cornutus (Clerck, 1758) en Fernández Galiano (1910) ya que la presencia de esta especie en la Península Ibérica se considera poco probable, habiéndose confundido seguramente con otras especies del género (Morano, 2000). La cita de Xyticus lanio que aparece en JiménezValverde (2002) es un error de identificación y corresponde, probablemente, a un ejemplar de Xysticus kochi, aunque el estado del ejemplar no permite asegurar la identificación.

Comentarios a los mapas de distribución conocida, distribución potencial y mapa de riqueza

Considerando las 110 cuadrículas UTM de 100 $\mathrm{km}^{2}$ de la Comunidad de Madrid, el número de registros es considerablemente bajo (Figs. 1 y 3 ). De las 73 especies, 20 cuentan únicamente con una cita de $10 \mathrm{x} 10 \mathrm{~km}$ (3 araneidos y 17 tomísidos) y especies de amplia distribución como M. acalypha, A. redii, A. cucurbitina o $S$. globosum cuentan con tan sólo 23, 18, 24 y 22 citas, respectivamente. La falta de registros es especialmente llamativa en el caso de los tomísidos, probablemente propiciado por su carácter críptico y, por tanto, por su difícil localización y captura.

Los mapas de distribución potencial de las especies aquí presentados (Figs. 1 y 3 ) deben considerarse aproximaciones preliminares. Las predicciones efectuadas mediante esta técnica se ven afectadas por dos parámetros fundamentales: el número y cobertura ambiental de los registros, y el número de variables predictoras (ver Beaumont et al., 2005). El número de registros y, más concretamente, su capacidad para representar la variedad de condiciones ambientales tolerada por las especies, determinará el rango ambiental dentro del cual existen condiciones potencialmente aptas para la presencia de la especie. De este modo las estimaciones de distribución potencial de aquellas especies infrarepresentadas serán siempre menores que la realidad y, dada la escasez de datos faunísticos en estos grupos, podemos asegurar que los mapas de distribución reales serán mayores que los mostrados. Este es especialmente el caso de las especies de la familia Thomisidae, cuyos mapas de distribución potencial y de riqueza de especies deben considerarse subestimaciones de las distribuciones reales.

A pesar de estos inconvenientes, consideramos que los mapas generales de distribución de la riqueza (Figs. 2 y 4 ) tienen altas probabilidades de reflejar el patrón de variación real. En ambas familias la Rampa, el área de transición entre la Sierra y las llanuras del Tajo, muestra la mayor riqueza de especies, ubicándose las zonas más pobres en los enclaves serranos de mayor altitud y en los suelos sedimentarios del sur de la Comunidad. En la Rampa madrileña está representado, prácticamente en su totalidad, el piso mesomediterráneo, con el encinar como serie de vegetación típica. Encinares, jarales y otras etapas degradativas del monte mediterráneo son hábitats con una alta complejidad estructural en los estratos arbustivo y subarbustivo. Esta complejidad del hábitat es el principal factor determinante de la riqueza de araneidos y tomísidos (JiménezValverde \& Lobo, en preparación), por lo que el gradiente de riqueza que surge en los mapas de este trabajo tiene un fuerte apoyo en base a los conocimientos ecológicos que tenemos de las dos familias de arañas. 


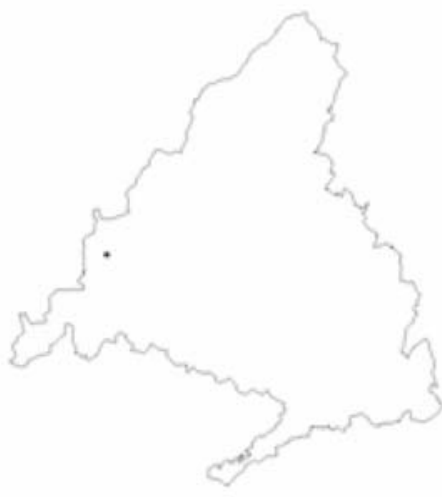

Coriarachne sp.

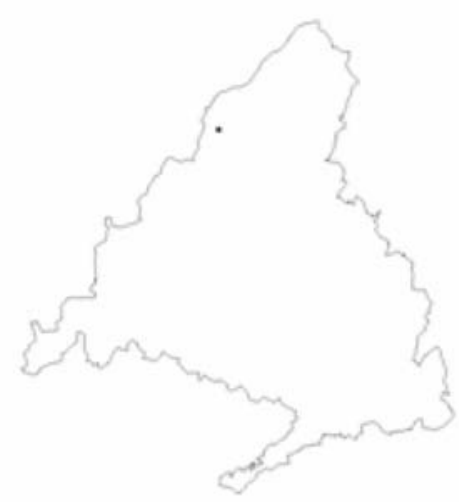

Diaea dorsata

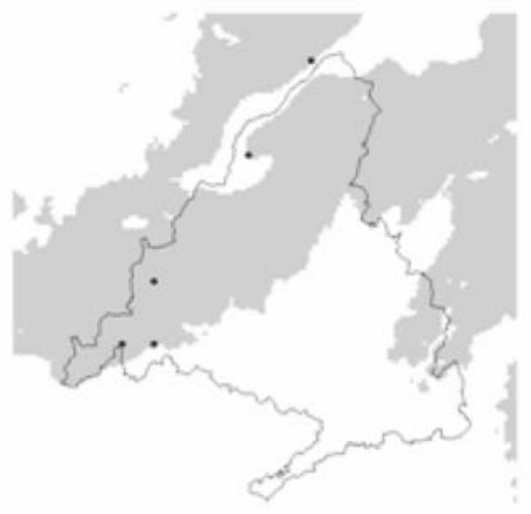

Misumena vatia

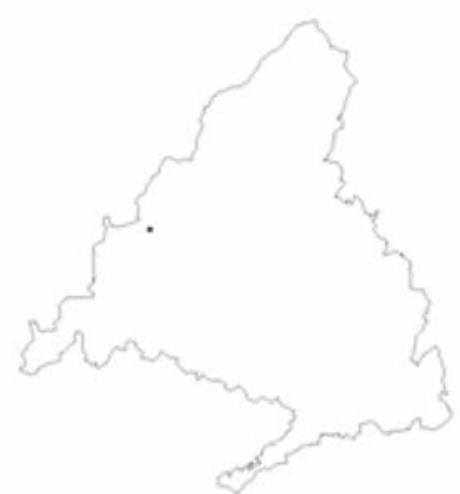

Firmicinus bivittatus

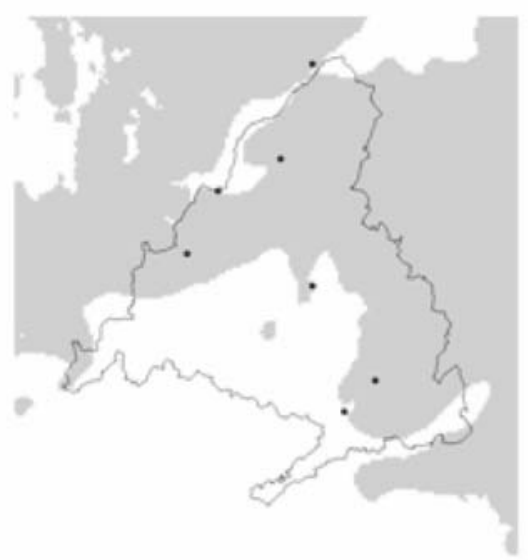

Heriaus mellotei

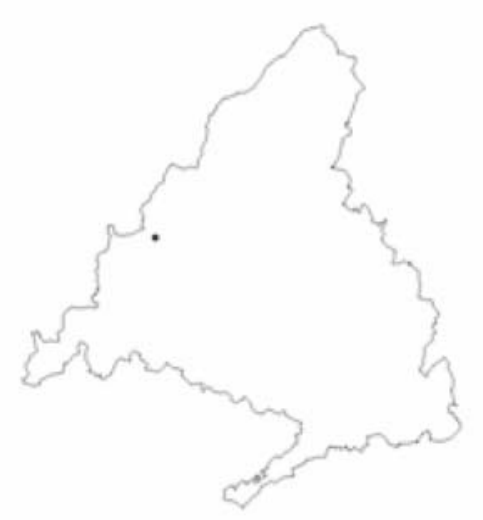

Misumenops sp.

Fig. 3.- Mapas de distribución conocida y potencial de las especies de la familia Thomisidae de la Comunidad de Madrid.

Fig. 3.- Known and potential distribution maps of species of the family Thomisidae in Comunidad de Madrid. 


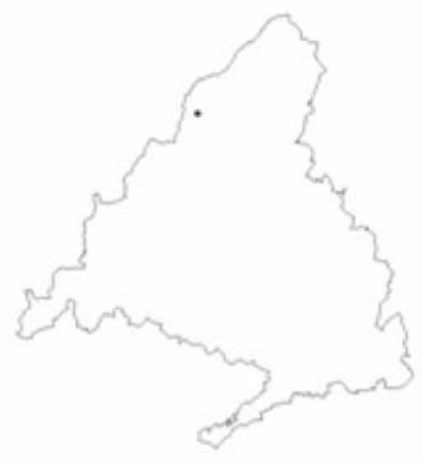

Ozyptilla atomaria

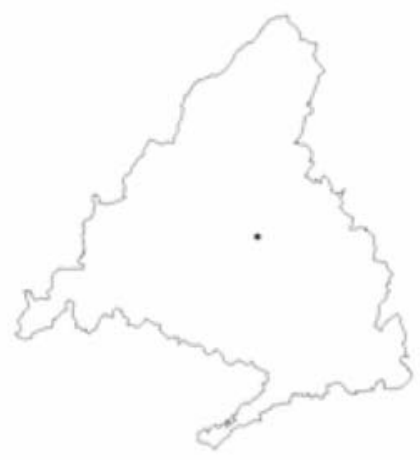

Ozyptilla blackwalli

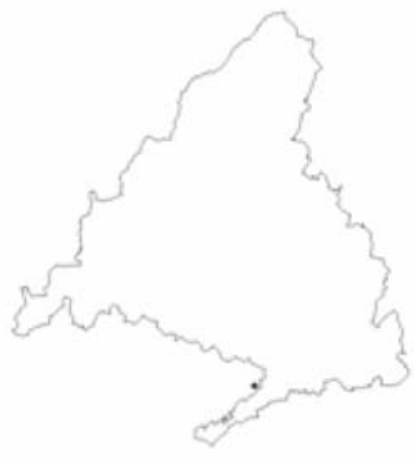

Ozyptilla perplexa

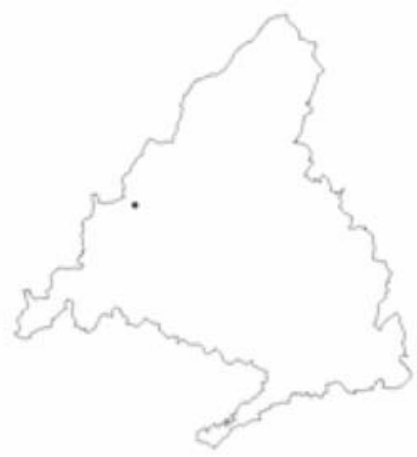

Ozyptilla bicuspis

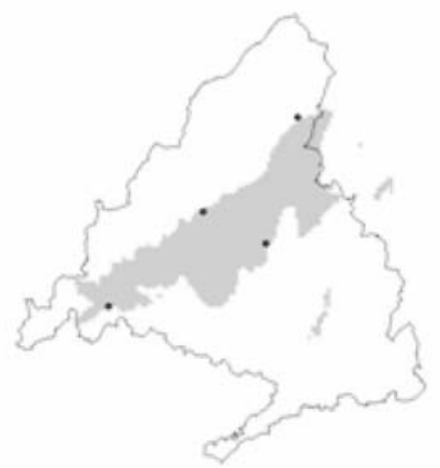

Ozyptilla pauxilla

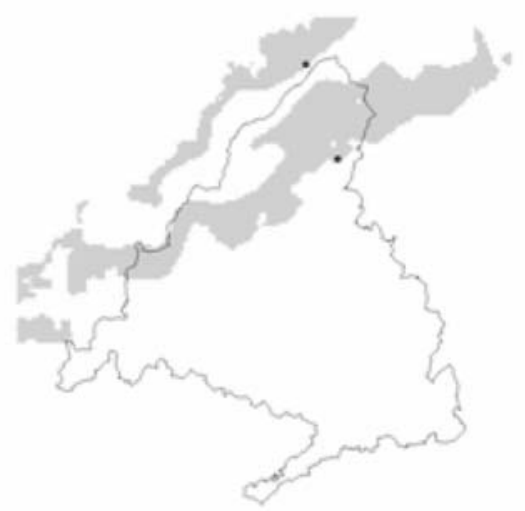

Ozyptilla umbraculorum 


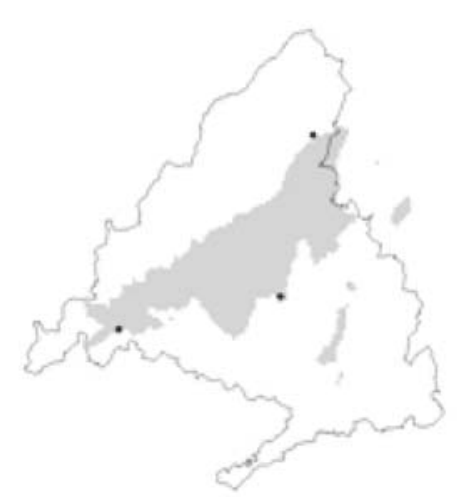

Pistus triuncatus

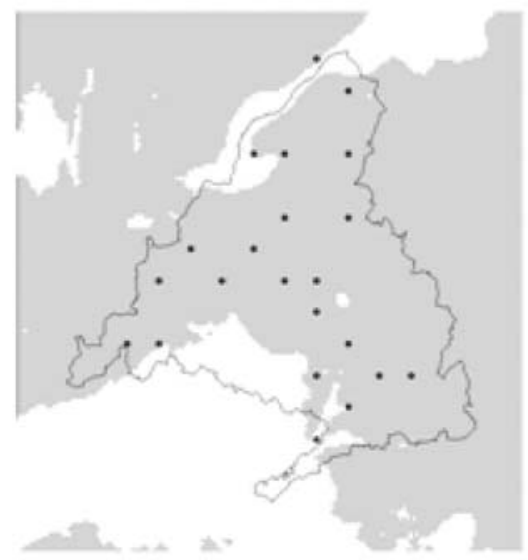

Synaema globosum

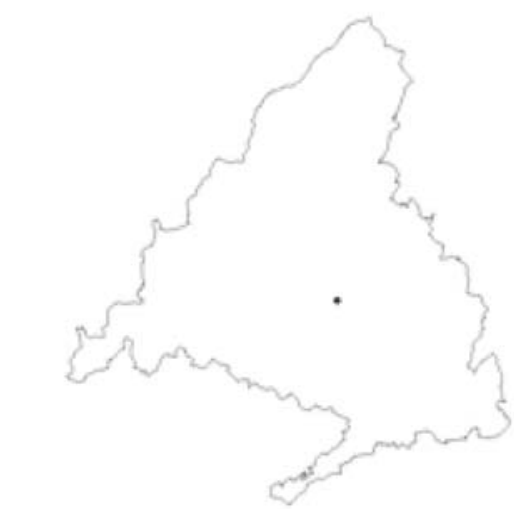

Tmarus piochardi

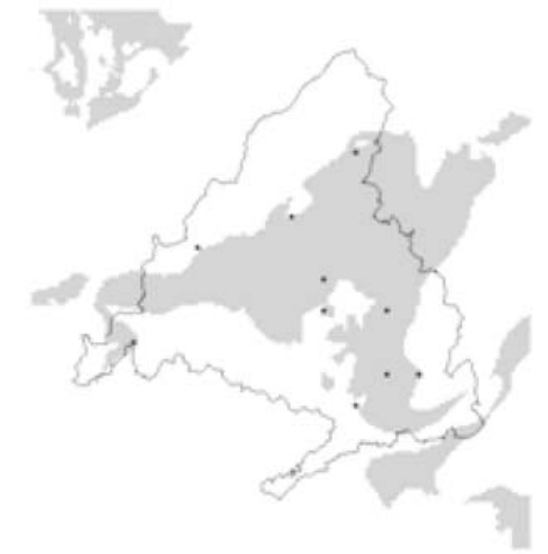

Runcinia grammica

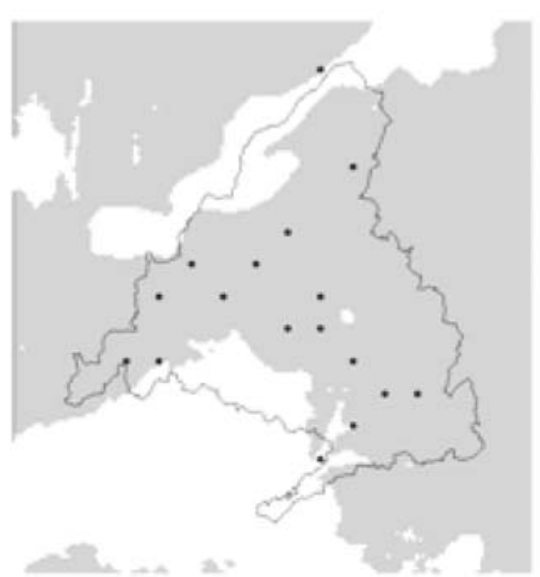

Thomisus onustus

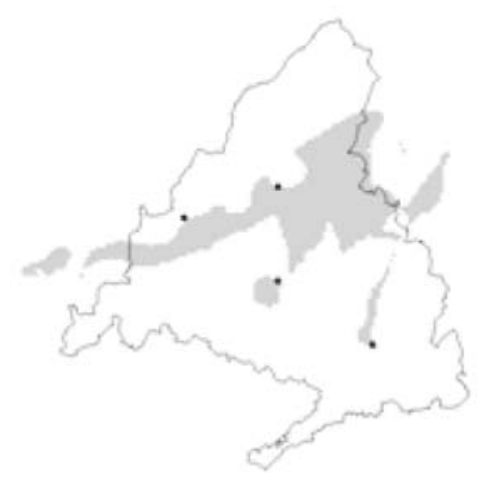

Tmarus staintoni

Fig. 3.- Cont. 


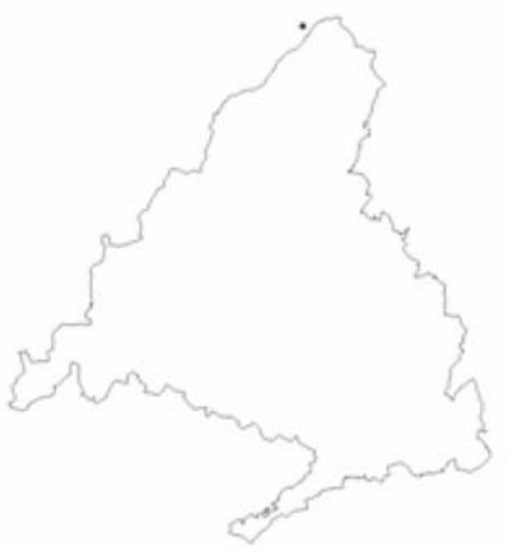

Tmarus stellio

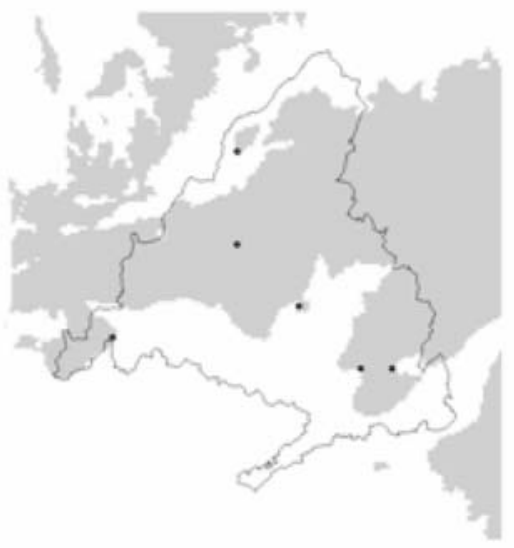

Xysticus audax

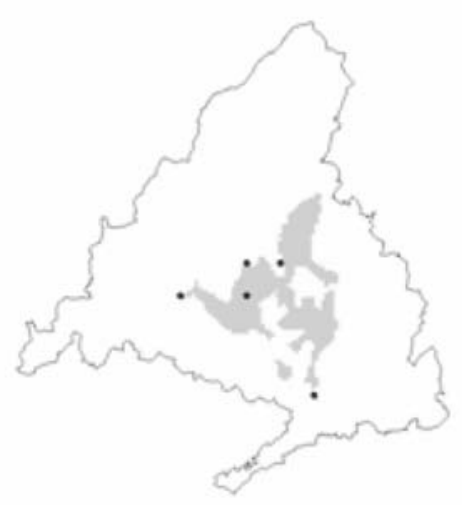

Xysticus bliteus

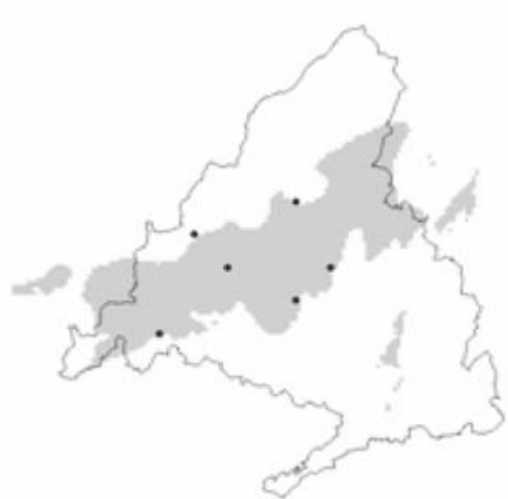

Xysticus acerbus

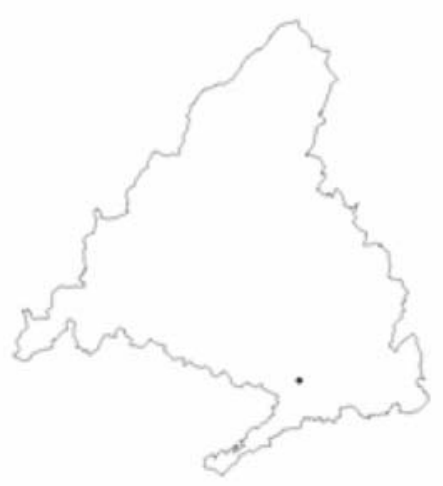

Xysticus bifasciatus

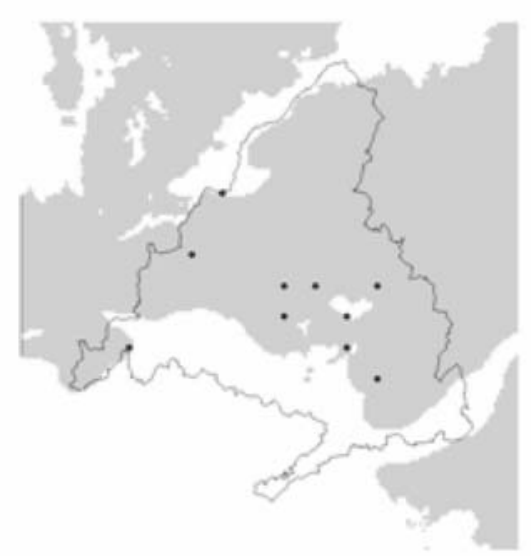

Xysticus bufo

Fig. 3.- Cont. 


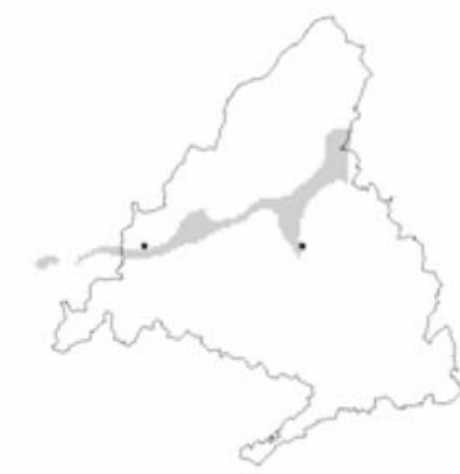

Xysticus caperatus

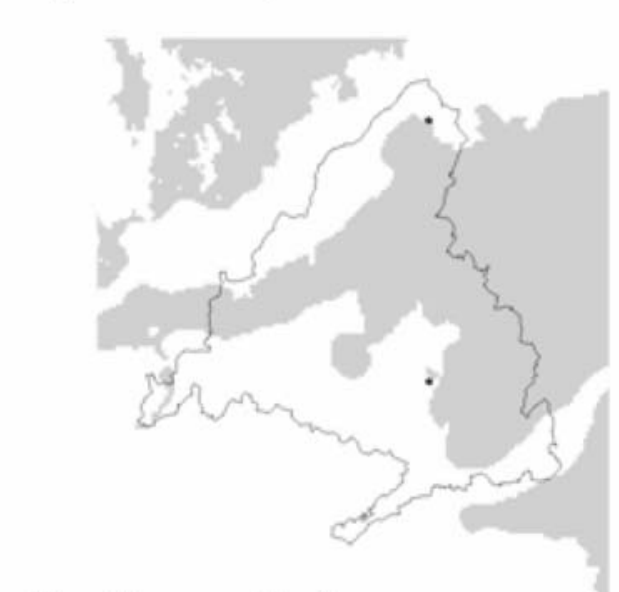

Xysticus cribatus

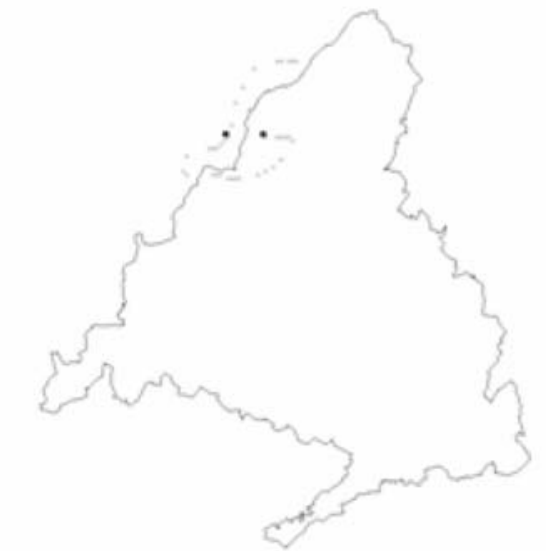

Xysticus ematicus

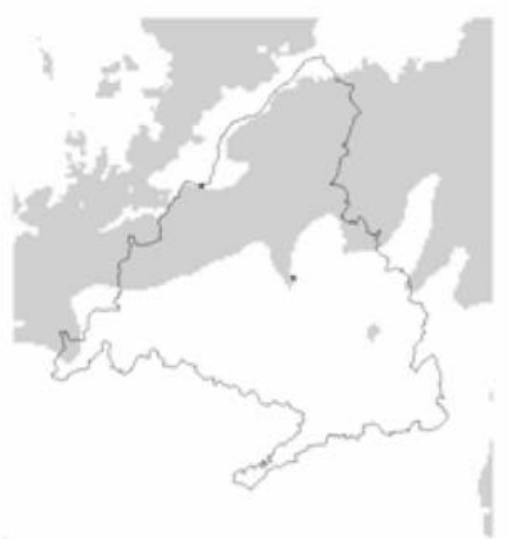

Xysticus cor

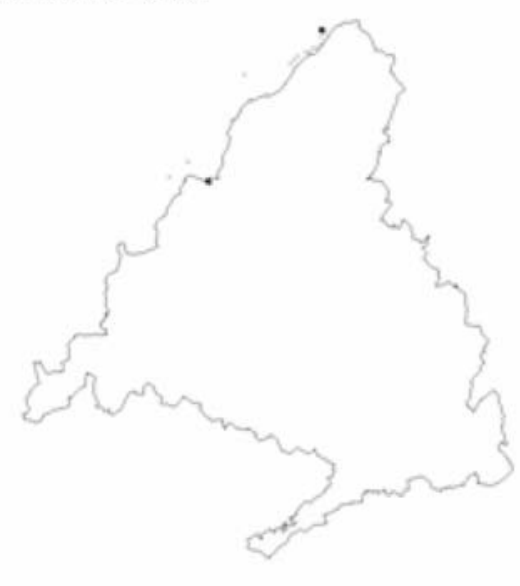

Xysticus cristatus

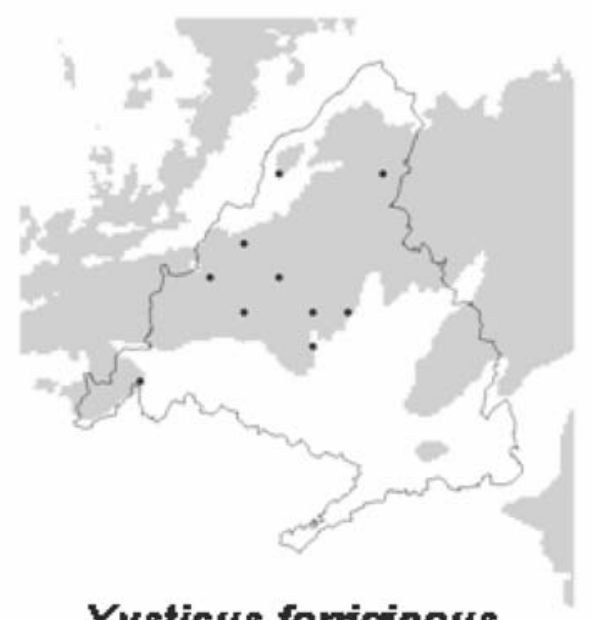

Xysticus femigineus

Fig. 3.- Cont. 


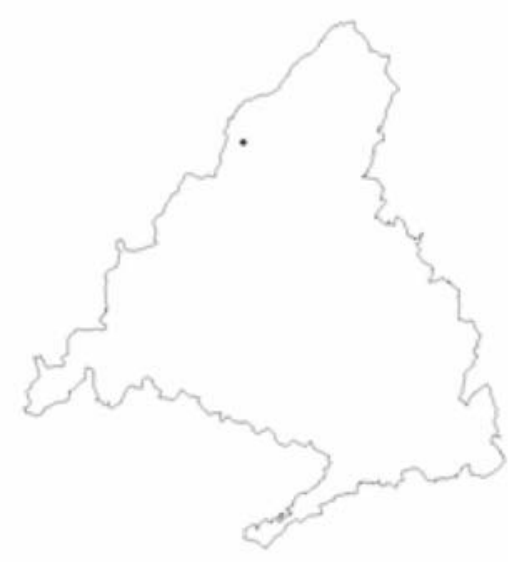

Xysticus gallicus

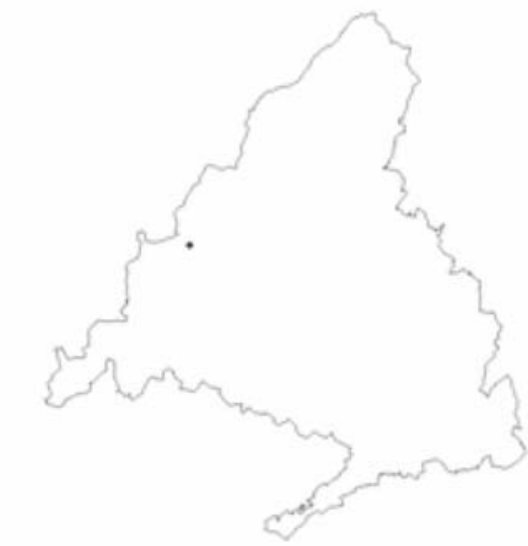

Xysticus kempelini

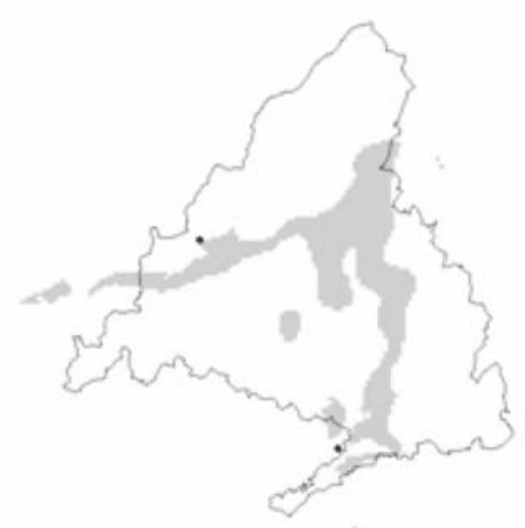

Xysticus lanio

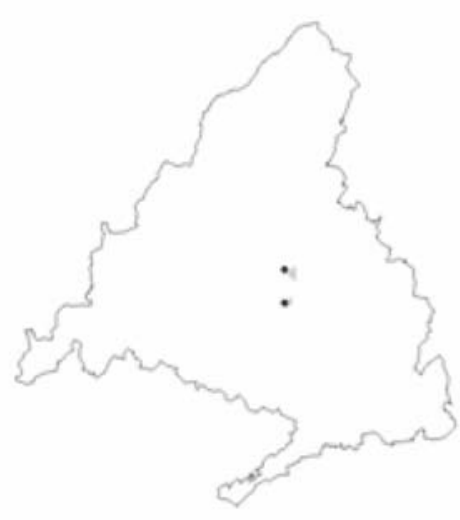

Xysticus grallator

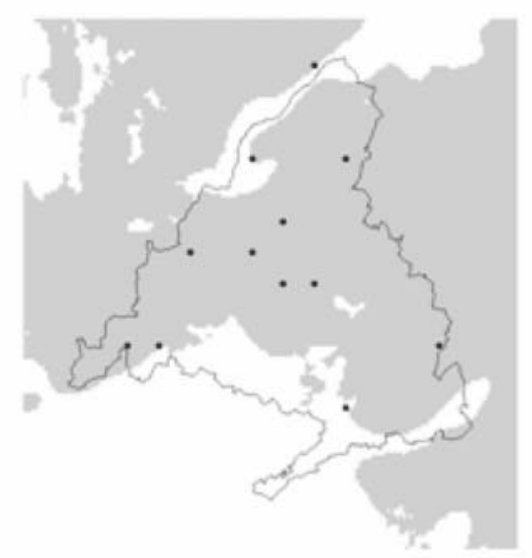

Xysticus kochi

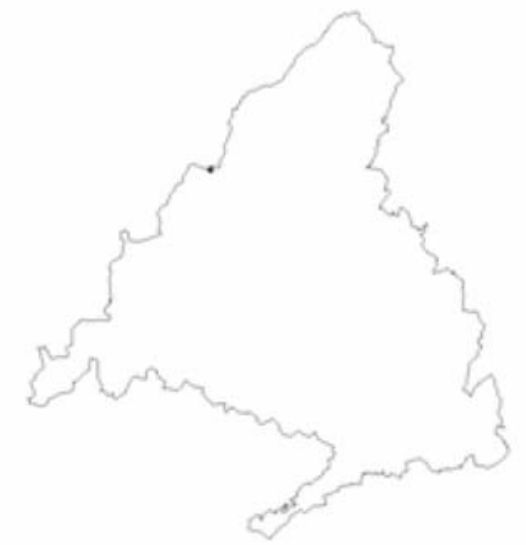

Xysticus lineatus

Fig. 3.- Cont. 


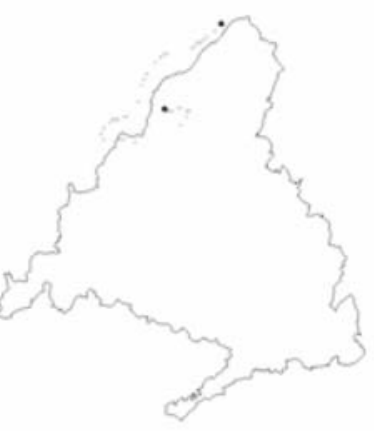

Xysticus ninni

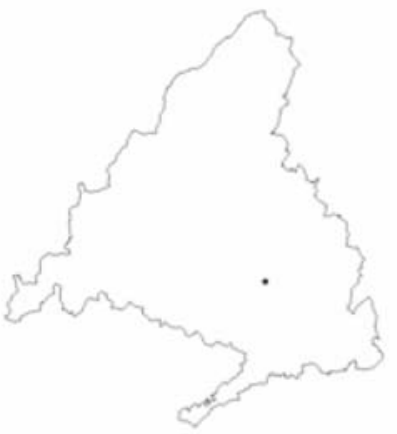

Xysticus ovatus

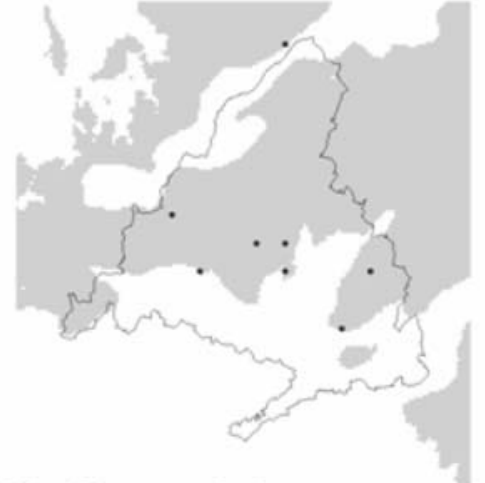

Xysticus sabulosus

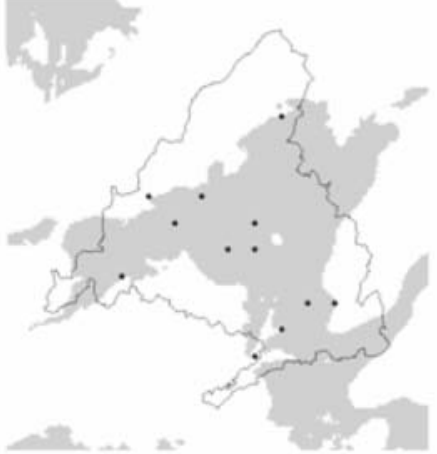

Xysticus nubilus

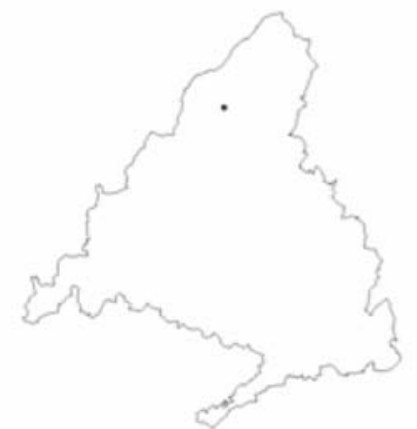

Xysticus robustus

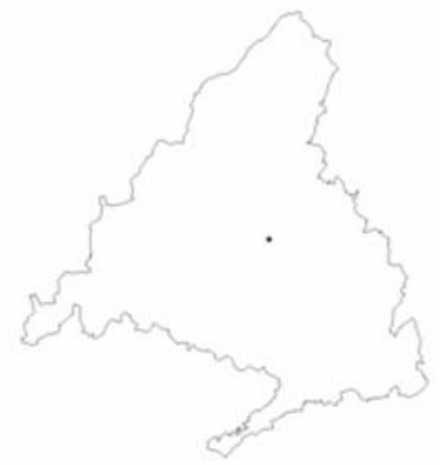

Xysticus semicarinatus

Fig. 3.-Fin Xysticus ulmi 


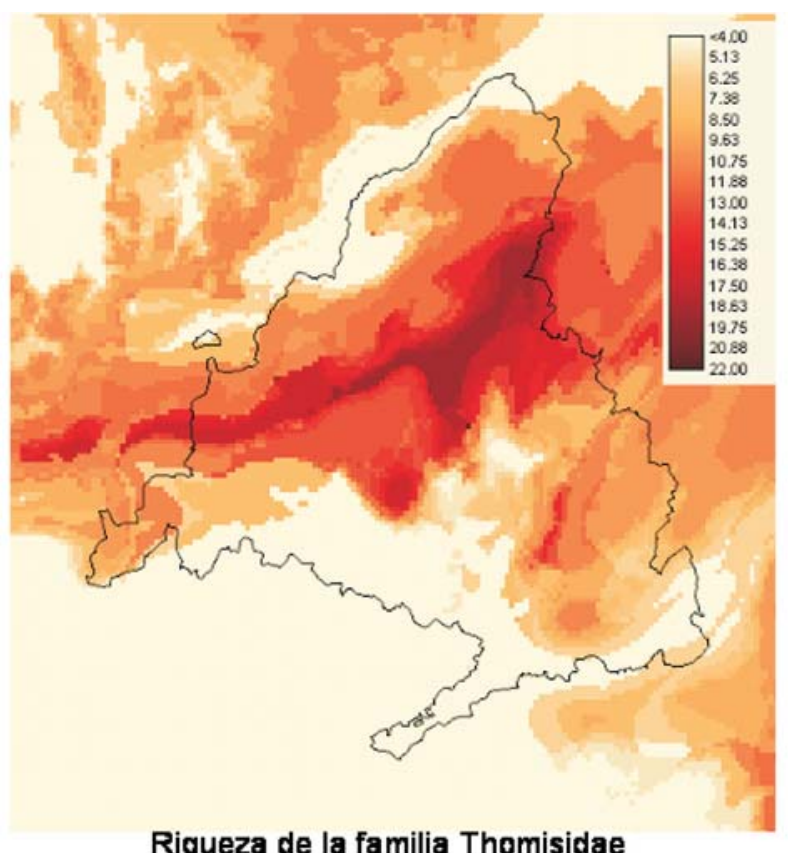

Riqueza de la familia Thomisidae

Fig. 4.- Mapa de riqueza predicha de la familia Thomisidae en la Comunidad de Madrid. Este mapa es el resultado de la suma de los mapas potenciales individuales de distribución.

Fig. 4.- Predicted species richness of the family Thomisidae in Comunidad de Madrid, calculated as the sum of individual potential distribution maps.

La baja diversidad de las altas cumbres de la Sierra puede deberse a tres motivos principales. Los dos primeros han de considerarse artefactos de muestreo. Es decir, por una parte faltan registros en estas zonas de difícil accesibilidad y, por otro, especies propias de estos enclaves no cuentan con el suficiente número de citas como para perfilar adecuadamente su nicho potencial (por ejemplo, muchas especies de Thomisidae, principalmente en los géneros Ozyptila y Xysticus; ver mapas). Estos dos factores hacen que la riqueza específica de las grandes alturas de la Sierra esté subestimada. Sin embargo, las altas cumbres suelen presentar una simplicidad en la estructura de la vegetación que nos hace pensar que, a pesar de los artefactos que hacen subestimar la riqueza, el patrón general puede aproximarse bien a la realidad; muestreos adicionales en estas zonas de gran altura ayudarán a corroborar este patrón.

El sur de la Comunidad, área de expansión de la ciudad de Madrid, cuenta con un suelo altamente urbanizado y un paisaje dominado por cultivos de secano y eriales periurbanos. Es una zona donde se aprecia una ausencia casi completa de registros de especies, quizás debido a la falta de atractivo que tiene este lugar para los entomólogos. Esto nos podría inducir a pensar que la baja diversidad de esta zona es un artefacto debido a sesgos en los muestreos, apreciables en gran variedad de taxones y áreas geográficas (Dennis \& Thomas, 2000; Reddy \& Dávalos, 2003; Romo \& García-Barros, 2005), aunque probablemente sea consecuencia de la pérdida de los hábitats naturales. En todo caso, serán necesarias más colectas en zonas poco alteradas antrópicamente de los términos del sur de Madrid.

\section{Conclusiones generales}

Aunque el listado de las familias Araneidae y Thomisidae para la Comunidad de Madrid puede considerarse bastante completo tras esta revisión, no ocurre lo mismo con el conocimiento corológico que disponemos para cada una de las especies. Este conocimiento es básico para poder elaborar planes de conservación eficaces y para poder evaluar el impacto de las actividades antrópicas sobre la diversidad biológica. A pesar de la alarmante ausencia de información, empleando una técnica de estimación sencilla hemos podido esbozar un primer patrón preliminar sobre la distribución espacial de la riqueza para cada una de las dos familias de arañas. En este patrón resalta la riqueza de la Rampa madrileña y la pobreza del sur de Madrid, muy alterado por la presión antrópica. La generación de modelos predictivos debe considerarse un proceso continuo de retroalimentación, en el que los primeros mapas ayudan a perfilar un primer patrón y a identificar zonas que necesitan datos faunísticos. Una vez conseguidos éstos, los mapas predictivos han de actualizarse y el proceso repetirse a fin de mejorar paulatinamente nuestra representación geográfica de la diversidad biológica.

\section{AGRADECIMIENTOS}

La Dra. Carmen Urones identificó amablemente algunos ejemplares de tomísidos. Este trabajo ha sido parcialmente financiado por los proyectos 07M/0080/2002 y GR/AMB/0750/2004 Consejería de Educación y Ciencia de la Comunidad de Madrid. El primer autor ha podido realizar este trabajo gracias a una beca predoctoral Museo Nacional de Ciencias Naturales/CSIC/ Comunidad de Madrid. 


\section{Referencias}

Beaumont, L. J., Hughes, L. \& Poulsen, M., 2005. Predicting species distributions: use of climatic parameters in BIOCLIM and its impact on predictions of species' current and future distributions. Ecological Modelling, 186: 250-269.

BusBy, J. R., 1986. A biogeoclimatic analysis of Notophagus cunninghamii (Hook.) Oerst. in southeastern Australia. Australian Journal of Ecology, 11: 1-7

Camargo, M. F., 1982. Estudio de los Araneidos (Araneae) de Valdelatas-Cantoblanco (Madrid). Tesis de Licenciatura, Facultad de Ciencias, Universidad Autónoma de Madrid. Inédita.

CArdoso, P., 2000. Portuguese spiders (Araneae): a preliminary checklist. Ekológica (Bratislava), 19(supl. 3): 19-29.

ClARK LABS., 2003. Idrisi Kilimanjaro. GIS software package. Clark Labs, Worcester, MA.

Coddington, J. A. \& Levi, H. W., 1991. Systematics and evolution of spiders. Annual Review of Ecology and Systematics, 22: 565-592.

De Castro, A. \& Alberdi, J. M., 2002. New spider species (Araneae) for the Spanish and Iberian fauna found in the Basque Country (Northern Spain). Munibe, 53: 175-181.

Dennis, R. L. H. \& Thomas, C. D., 2000. Bias in butterfly distribution maps: the influence of hot spots and recorder's home range. Journal of Insect Conservation, 4: 73-77.

Fernández Galiano, E., 1910. Datos para el conocimiento de la distribución geográfica de los Arácnidos de España. Memorias de la Real Sociedad española de Historia Natural, 6: 1-86.

FoelIX, R. F., 1996. Biology of spiders. Oxford University Press. New York. 330 pp.

JimÉnEZ-VAlVerde, A., 2002. Presencia en la Comunidad de Madrid (España central) del endemismo ibérico Ozyptila umbraculorum Simon, 1832 (Araneae, Thomisidae). Revista Ibérica de Aracnología, 6: 225-227.

JimÉneZ-VAlverde, A., 2005. Contribución al conocimiento de los saltícidos (Araneae, Salticidae) de la Comunidad de Madrid (España central). Boletín de la Sociedad Entomológica Aragonesa, 37: 289-296.

Jiménez-Valverde, A., Barriga Bernal，J. C. \& Morano, E., 2004. Datos interesantes sobre la distribución de Araniella opisthographa (Kulczynski, 1905) у A. inconspicua (Simon, 1874) (Araneae: Araneidae) en la Península Ibérica. Revista Ibérica de Aracnología, 9: 269-270.
JiMÉNEZ-VALVERDE, A. \& LoBO, J. M., 2004. Un método sencillo para seleccionar puntos de muestreo con el objeto de inventariar taxones hiperdiversos: el caso práctico de las familias Araneidae y Thomisidae (Araneae) en la Comunidad de Madrid (España). Ecología, 18: 297-308

JimÉNEZ-VALVERDE, A. \& LoBO, J. M., 2005. Determining a combined sampling procedure for a reliable estimation of Araneidae and Thomisidae assemblages (Arachnida: Araneae). Journal of Arachnology, 33: 33-42.

Marc, P., Canard, A. \& Ysnel, F., 1999. Spiders (Araneae) useful for pest limitation and bioindication. Agriculture, Ecosystems and Environment, 74: 229-273.

Melic, A., 2001. Arañas endémicas de la Península Ibérica e Islas Baleares (Arachnida: Araneae). Revista Ibérica de Aracnología, 4: 35-92.

Morano, E., 2000. Las especies de Larinioides Caporiacco, 1934 (Araneae, Araneidae) de la Península Ibérica. Revista Ibérica de Aracnología, 5: 67-74.

MorAnO, E., 2004. Introducción a la diversidad de las arañas Iberobaleares. Munibe, (suplemento) 21: 92137.

Platnick, N. I., 2005. The World Spider Catalog v 5.5. American Museum of Natural History, en http://research.amnh.org/entomology/spiders/catalog/INTRO1 .html

RedDY, S. \& DÁvalos, L. M., 2003. Geographical sampling bias and its implications for conservation priorities in Africa. Journal of Biogeography, 30: 1719-1727.

Romo, H. \& García-Barros, E., 2005. Distribución e intensidad de los estudios faunísticos sobre mariposas diurnas en la Península Ibérica e Islas Baleares (Lepidoptera, Papilionoidea y Hesperioidea). Graellsia, 81(1): 37-50.

Simon, E., 1932. Les Arachnides de France. Tome VI. Encyclopédie Loret et L. Mulo. Paris. 978 pp.

Wise, D. H., 1993. Spiders in Ecological Webs. Cambridge University Press. Cambridge. 328 pp. 
Apéndice 1.- Coordenadas UTM 10 x $10 \mathrm{~km}$ para cada especie de las familias Araneidae y Thomisidae en la Comunidad de Madrid.

Appendix 1.- UTM 10x10 km coordinates for each species of families Araneidae and Thomisidae in Comunidad de Madrid.

\begin{tabular}{|c|c|c|c|}
\hline \multicolumn{2}{|c|}{ ARANEIDAE } & & 30TVL33 \\
\hline \multirow{15}{*}{ Aculepeira armida } & & & 30TVL41 \\
\hline & 30TUK/5 & Araniella alpica & $30 \mathrm{TVK} 09$ \\
\hline & 301UK97 & & 30TVL11 \\
\hline & 30TUK98 & & 30TVL12 \\
\hline & 30TVK09 & & 30 TVL22 \\
\hline & 30 TVK 29 & Araniella cucurbitina & 30TUK98 \\
\hline & 30TVK43 & & 30TVK09 \\
\hline & 30 TVK 47 & & 30 TVK 18 \\
\hline & 30TVK49 & & $30 \mathrm{TVK} 26$ \\
\hline & 30TVK54 & & 30TVK38 \\
\hline & 30TVK66 & & $30 \mathrm{TVK} 47$ \\
\hline & 30 TVL20 & & $30 \mathrm{TVK} 48$ \\
\hline & 30TVL31 & & 30 TVK 49 \\
\hline & 30 TVL43 & & 30 TVK 65 \\
\hline & 30TVL52 & & 30 TVK 75 \\
\hline \multirow[t]{7}{*}{ Aculepeira ceropegia } & 30TUK98 & & 30 TVL 10 \\
\hline & 30TVK09 & & S0TVI 11 \\
\hline & 30 TVL10 & & $301 \mathrm{VLI}$ \\
\hline & 30 TVL11 & & 30TVL21 \\
\hline & & & 30TVL22 \\
\hline & $30 \mathrm{TVL} 23$ & & 30TVL23 \\
\hline & 30TVL32 & & 30TVL30 \\
\hline \multirow[t]{18}{*}{ Agalenatea redii } & 30TUK86 & & 30TVL31 \\
\hline & 30TVK09 & & 30TVL32 \\
\hline & 30TVK18 & & 30 TVL 33 \\
\hline & 30 TVK26 & & 30TVL41 \\
\hline & 30 TVK29 & & 30TVL42 \\
\hline & $30 \mathrm{TVK} 38$ & & 30 TVL43 \\
\hline & 30 TVK43 & & 30 TVL45 \\
\hline & 30TVK48 & & 30TVL52 \\
\hline & 30TVK54 & Araniella inconspicua & 30 TVK 29 \\
\hline & 30 TVK56 & Araniella opisthographa & 30 TVL11 \\
\hline & 30TVK65 & & 30TVL22 \\
\hline & 30 TVK 75 & & 30 TVL30 \\
\hline & 30TVL22 & & 30TVL32 \\
\hline & 30 TVL30 & & 30 TVL45 \\
\hline & 30TVL42 & & 30TVL52 \\
\hline & 30TVL43 & Argiope bruennichi & 30TVK09 \\
\hline & 30TVL50 & & $30 \mathrm{TVK} 16$ \\
\hline & 30 TVL52 & & 30 TVK 29 \\
\hline \multirow[t]{8}{*}{ Araneus angulatus } & 30TUK86 & & $30 \mathrm{TVK} 36$ \\
\hline & 30TUK98 & & $30 \mathrm{TVK} 37$ \\
\hline & 30TVK09 & & 30 TVK 38 \\
\hline & 30 TVK 49 & & 30 TVK47 \\
\hline & 30TVL01 & & $30 \mathrm{TVK} 48$ \\
\hline & 30 TVL10 & & $30 \mathrm{TVK} 54$ \\
\hline & 30TVL11 & & 30 TVK57 \\
\hline & 30TVL31 & & 30 TVK 66 \\
\hline \multirow[t]{3}{*}{ Araneus diadematus } & 30 TVK45 & & 30TVL42 \\
\hline & 30TVL11 & Argiope lobata & 30TUK75 \\
\hline & 30TVL32 & & $30 \mathrm{TVK} 36$ \\
\hline \multirow[t]{9}{*}{ Araneus pallidus } & 30TUK98 & & $30 \mathrm{TVK} 47$ \\
\hline & 30TVK29 & & $30 \mathrm{TVK} 49$ \\
\hline & $30 \mathrm{TVK} 36$ & & 30 TVK54 \\
\hline & $30 \mathrm{TVK} 37$ & & $30 \mathrm{TVK} 56$ \\
\hline & 30 TVK 43 & & 30 TVK57 \\
\hline & 30 TVK 47 & & 30 TVK67 \\
\hline & 30TVK48 & Atea sturmi & 30TVL01 \\
\hline & 30 TVK 49 & Cercidia prominens & 30TVL32 \\
\hline & 30TVK65 & Cyclosa algerica & 30TUK86 \\
\hline
\end{tabular}

\begin{tabular}{|c|c|c|c|}
\hline & 30TUK96 & & $30 \mathrm{TVK} 54$ \\
\hline & 30TUK98 & & 30 TVK 57 \\
\hline & 30TVK18 & & 30 TVK 75 \\
\hline & 30TVK38 & & 30 TVK 86 \\
\hline & 30 TVK 48 & & 30 TVL10 \\
\hline & 30TVK49 & & 30TVK19 \\
\hline & $30 \mathrm{TVK} 75$ & & 30TVL20 \\
\hline & 30TVL30 & & 30TVL21 \\
\hline & 30TVL41 & & 30 TVL30 \\
\hline & 30TVL45 & & 30TVL41 \\
\hline & 30TVL52 & & 30TVL42 \\
\hline Cyclosa conica & 30 TUK 86 & & 30 TVL52 \\
\hline & 30TVK09 & Larinioides suspicax & 30 TVK 38 \\
\hline & 30TVK29 & & 30TVL41 \\
\hline & 30TVK43 & Mangora acalypha & 30 TUK 86 \\
\hline & $30 \mathrm{TVK} 47$ & & 30TUK96 \\
\hline & 30TVL11 & & 30TUK98 \\
\hline & 30TVL21 & & 30TVK09 \\
\hline & 30 TVL 22 & & 30 TVK 18 \\
\hline & 30TVL23 & & 30 TVK 29 \\
\hline & 30 TVL50 & & 30 TVK 37 \\
\hline & 30TVL52 & & 30 TVK 38 \\
\hline Cyclosa insulana & $30 \mathrm{TVK} 45$ & & $30 \mathrm{TVK} 43$ \\
\hline & 30 TVK56 & & 30 TVK 48 \\
\hline Gibbaranea bituberculata & 30TVK09 & & 30TVK49 \\
\hline & 30TVK 18 & & 30 TVK54 \\
\hline & 30TVK29 & & 30 TVK 56 \\
\hline & $30 \mathrm{TVK} 38$ & & 30 TVK75 \\
\hline & $30 \mathrm{TVK} 43$ & & 30TVL20 \\
\hline & 30TVK48 & & 30TVL21 \\
\hline & 30TVK54 & & 30 TVL22 \\
\hline & 30TVK58 & & 30 TVL23 \\
\hline & 30TVL41 & & 30 TVL30 \\
\hline & 30TVL52 & & 30 TVL32 \\
\hline Gibbaranea gibbosa & 30TUK96 & & 30TVL41 \\
\hline & $30 \mathrm{TVK} 47$ & & 30 TVL45 \\
\hline & 30TVL22 & & 30TVL52 \\
\hline Hyposinga pygmaea & 30TVK45 & Neoscana subfusca & 30TUK96 \\
\hline & 30 TVL32 & & 30 TVK 45 \\
\hline Hyposonga albovittata & 30 TUK96 & & 30 TVK 65 \\
\hline & 30TVK09 & Neoscona adianta & 30TUK99 \\
\hline & 30TVK18 & & 30 TVK 29 \\
\hline & 30 TVK 29 & & 30 TVK 38 \\
\hline & $30 \mathrm{TVK} 48$ & & 30 TVK 48 \\
\hline & 30TVK49 & & 30TVK49 \\
\hline & 30 TVK54 & & 30 TVK 54 \\
\hline & 30 TVK 65 & & 30 TVK 56 \\
\hline & 30 TVK 78 & & 30TVK64 \\
\hline & 30 TVL 22 & & 30 TVK 65 \\
\hline & 30TVL30 & & 30TVK19 \\
\hline & 30TVL42 & & 30TVL21 \\
\hline & 30TVL52 & & 30 TVL22 \\
\hline & 30 TVK54 & & 30 TVL30 \\
\hline & 30TVL32 & & 30TVL31 \\
\hline Larinioides sclopetarius & 30TUK99 & & 30 TVL32 \\
\hline & 30TVK09 & & 30TVL41 \\
\hline & $30 \mathrm{TVK} 18$ & & 30 TVL45 \\
\hline & 30TVK29 & & 30TVL52 \\
\hline & 30 TVK38 & Singa hamata & 30 TVK 54 \\
\hline & $30 \mathrm{TVK} 47$ & Zilla diodia & 30 TUK86 \\
\hline
\end{tabular}




$\begin{array}{cc} & \text { 30TUK96 } \\ & \text { 30TVK09 } \\ & \text { 30TVK29 } \\ & \text { 30TVK43 } \\ & \text { 30TVK48 } \\ & \text { 30TVK65 } \\ & \text { 30TVL23 } \\ & \text { 30TVL30 } \\ & \text { 30TVL32 } \\ & \text { 30TVL41 } \\ & \text { 30TVL45 } \\ & \text { 30TVL01 } \\ \text { Zygiella x-notata } & \text { 30TV65 } \\ & \text { 30TVL42 }\end{array}$

\section{THOMISIDAE}

$\begin{array}{ll}\text { Coriarachne sp. } & \text { 30TUK98 } \\ \text { Diaea dorsata } & \text { 30TVL22 } \\ \text { Firmicinus bivittatus } & \text { 30TVK09 } \\ \text { Heriaeus mellotei } & \text { 30TVK09 } \\ & \text { 30TVK48 } \\ & \text { 30TVK54 } \\ & \text { 30TVK65 } \\ & \text { 30TVL11 } \\ & \text { 30TVL32 } \\ & \text { 30TVL45 } \\ & \text { 30TUK86 } \\ & \text { 30TUK96 } \\ & \text { 30TUK98 } \\ \text { Misumena vatia } & \text { 30TVL22 } \\ & \text { 30TVL45 } \\ & \text { 30TVK09 } \\ & \text { 30TVL22 } \\ \text { Misumenops sp. } & \text { 30TVK48 } \\ \text { Ozyptila atomaria } & \text { 30TVK48 } \\ \text { Ozyptila bicuspis } & \text { 30TVK56 } \\ \text { Ozyptila blackwalli } & \text { 30TUK96 } \\ & \text { 30TVK29 } \\ \text { Ozyptila pauxilla } & \text { 30TVK48 } \\ & \text { 30TVL52 } \\ \text { Runcinia grammica } & \text { 30TVK43 } \\ \text { Ozyptila perplexa } & \text { 30TVL45 } \\ \text { Ozyptila umbraculorum } & \text { 30TVL52 } \\ \text { Pistus truncatus } & \text { 30TUK96 } \\ & \text { 30TVK47 } \\ & \end{array}$

\begin{tabular}{|c|c|c|c|c|}
\hline 30TVK09 & Tmarus staintoni & 30TVK09 & & $30 \mathrm{TVK} 48$ \\
\hline 30TVK47 & & 30 TVK 37 & & 30 TVL10 \\
\hline 30TVK48 & & 30 TVK65 & & 30TVL22 \\
\hline 30TVK54 & & 30 TVL30 & & 30TVL52 \\
\hline 30TVK65 & Tmarus stellio & 30 TVL45 & Xysticus gallicus & 30TVL22 \\
\hline 30TVK67 & Xysticus acerbus & 30 TUK96 & Xysticus grallator & 30 TVK 47 \\
\hline $30 \mathrm{TVK} 75$ & & 30TVK09 & & $30 \mathrm{TVK} 48$ \\
\hline 30 TVL30 & & 30 TVK 18 & Xysticus kempelini & 30TVK09 \\
\hline 30TVL52 & & 30 TVK 37 & Xysticus kochi & 30 TUK86 \\
\hline 30TUK86 & & 30 TVK48 & & 30TUK96 \\
\hline 30TUK96 & & 30 TVL30 & & 30TVK09 \\
\hline 30TUK98 & Xysticus audax & 30TUK86 & & $30 \mathrm{TVK} 29$ \\
\hline 30TVK09 & & 30 TVK 29 & & 30 TVK38 \\
\hline 30TVK18 & & 30 TVK47 & & $30 \mathrm{TVK} 48$ \\
\hline 30TVK29 & & 30TVK65 & & 30 TVK54 \\
\hline 30TVK38 & & 30 TVK 75 & & 30 TVK86 \\
\hline 30TVK43 & & 30TVL22 & & 30TVL22 \\
\hline $30 \mathrm{TVK} 45$ & Xysticus bifasciatus & 30 TVK54 & & 30 TVL30 \\
\hline $30 \mathrm{TVK} 47$ & Xysticus bliteus & 30 TVK17 & & 30TVL45 \\
\hline 30TVK48 & & 30 TVK 37 & & 30TVL52 \\
\hline 30TVK54 & & 30 TVK 38 & Xysticus lanio & 30TVK09 \\
\hline 30 TVK56 & & 30 TVK48 & & 30 TVK43 \\
\hline 30TVK65 & & 30 TVK 54 & Xysticus lineatus & 30TVL11 \\
\hline 30TVK75 & Xysticus bufo & 30TUK86 & Xysticus ninni & 30TVL22 \\
\hline 30TVL22 & & 30TVK09 & & 30 TVL45 \\
\hline 30 TVL30 & & 30TVK37 & Xysticus nubilus & 30TUK96 \\
\hline 30TVL32 & & 30 TVK 38 & & 30TVK09 \\
\hline 30TVL45 & & 30 TVK 48 & & 30 TVK 18 \\
\hline 30 TVL50 & & 30 TVK 56 & & 30 TVK 29 \\
\hline 30TVL52 & & 30 TVK 57 & & 30 TVK 37 \\
\hline 30TVL54 & & 30 TVK 65 & & 30TVK43 \\
\hline 30TUK86 & & 30TVK68 & & $30 \mathrm{TVK} 47$ \\
\hline 30TUK96 & & 30TVL11 & & $30 \mathrm{TVK} 48$ \\
\hline 30TUK98 & Xysticus caperatus & 30TUK98 & & 30 TVK54 \\
\hline 30TVK09 & & 30TVK48 & & $30 \mathrm{TVK} 65$ \\
\hline 30TVK18 & Xysticus cor & 30TVK48 & & 30 TVK75 \\
\hline 30TVK29 & & 30TVL11 & & 30TVL52 \\
\hline 30 TVK37 & Xysticus cribatus & 30 TVK56 & Xysticus ovatus & 30 TVK56 \\
\hline 30TVK43 & & 30TVL54 & Xysticus robustus & 30TVL32 \\
\hline 30 TVK47 & Xysticus cristatus & 30TVL11 & Xysticus sabulosus & 30TVK09 \\
\hline 30TVK48 & & 30TVL45 & & 30 TVK 17 \\
\hline 30TVK54 & Xysticus erraticus & 30TVL12 & & $30 \mathrm{TVK} 38$ \\
\hline 30 TVK56 & & 30 TVL22 & & $30 \mathrm{TVK} 47$ \\
\hline 30TVK65 & Xysticus ferrugineus & 30 TUK 86 & & $30 \mathrm{TVK} 48$ \\
\hline 30 TVK75 & & 30TVK09 & & 30 TVK65 \\
\hline 30TVL30 & & 30TVK18 & & $30 \mathrm{TVK} 77$ \\
\hline 30TVL45 & & 30 TVK 29 & & 30TVL45 \\
\hline 30TVL52 & & 30 TVK 37 & Xysticus semicarinatus & 30TVL12 \\
\hline 30TVK47 & & 30 TVK 38 & Xysticus ulmi & $30 \mathrm{TVK} 48$ \\
\hline
\end{tabular}

